\title{
Influence of s-d interfacial scattering on the magnetoresistance of magnetic tunnel junctions
}

\author{
D. Bagrets \\ CEA/Grenoble, Département de Recherche Fondamentale sur la Matière Condensée, SP2M/NM, 38054 Grenoble, France and \\ Department of Physics, M. V. Lomonosov Moscow State University, 119899 Moscow, Russia \\ A. Bagrets \\ Department of Physics, M. V. Lomonosov Moscow State University, 119899 Moscow, Russia \\ A. Vedyayev \\ CEA/Grenoble, Département de Recherche Fondamentale sur la Matière Condensée, SP2M/NM, 38054 Grenoble, France and \\ Department of Physics, M. V. Lomonosov Moscow State University, 119899 Moscow, Russia \\ B. Dieny \\ CEA/Grenoble, Département de Recherche Fondamentale sur la Matière Condensée, SP2M/NM, 38054 Grenoble, France
}

(Dated: 22 August, 2001)

\begin{abstract}
We propose the two-band $s$ - $d$ model to describe theoretically a diffuse regime of the spin-dependent electron transport in magnetic tunnel junctions (MTJ's) of the form F/O/F where F's are $3 d$ transition metal ferromagnetic layers and $\mathrm{O}$ is the insulating spacer. We aim to explain the strong interface sensitivity of the tunneling properties of MTJ's and investigate the influence of electron scattering at the nonideal interfaces on the degradation of the TMR magnitude. The generalized Kubo formalism and the Green's functions method were used to calculate the conductance of the system. The vertex corrections to the conductivity were found with the use of "ladder" approximation combined with the coherent-potential approximation (CPA) that allowed to consider the case of strong electron scattering. It is shown that the Ward identity is satisfied in the framework of this approximation that provides the necessary condition for a conservation of a tunneling current. Based on the known results of ab-initio calculations of the TMR for ballistic junctions, we assume that exchange split quasi-free $s$-like electrons with the density of states being greater for the majority spin sub-band give the main contribution to the TMR effect. We show that, due to interfacial inter-band scattering, the TMR can be substantially reduced even down to zero value. This is related to the fact that delocalized quasi-free electrons can scatter into the strongly localized $d$ sub-band with the density of states at the Fermi energy being larger for minority spins compared to majority spins. It is also shown that spin-flip electron scattering on the surface magnons within the interface leads to a further decrease of the TMR at finite temperature.
\end{abstract}

PACS numbers: 75.70.-i, 73.40.Gk, 73.40.Rw, 85.30.Mn

\section{INTRODUCTION}

Magnetic tunnel junctions (MTJ's) consisting of two ferromagnetic layers separated by the insulating spacer exhibit the tunneling magnetoresistance effect (TMR) when they are switched by applying a magnetic field from the antiparallel to parallel alignment of magnetizations in magnetic layers. The TMR effect was first observed by M. Julliere in 1975- Julliere found that the tunneling conductance of the trilayer structure $\mathrm{Fe} / \mathrm{Ge} / \mathrm{Co}$ depended on the angle between magnetizations in the $\mathrm{Fe}$ and Co layers. The measured amplitude of the TMR in these experiments was $14 \%$ at $4.2 \mathrm{~K}$. Only 20 years later, the large values of TMR at room temperature were obtainer in magnetic junctions comprising the $\mathrm{Al}_{2} \mathrm{O}_{3}$ barrier2 s. $^{-}$. Since that time, there has been a renewed technological and fundamental interest to the tunneling phenomenon and during the last decade a lot of experimental and theoretical papers were published on this topic (see reviews $[5,6,7,8]$ ).

Experimentally it was observed 10 that the TMR de- pends critically on the material of the insulating barrier and on the conditions of its preparation, in particular on the imperfections of the interfaces between the metal and the insulating layer 10 . On the other hand, the first theory suggested by M. Julliere expresses the TMR ratio in terms of the effective spin polarizations $P_{1}$ and $P_{2}$ of two magnetic layers via the expression $\mathrm{TMR}=2 P_{1} P_{2} /\left(1+P_{1} P_{2}\right)$, and thus predicts no dependence of the TMR on the parameters of the barrier. In spite of Julliere's formula is widely used for the interpretation of the experimental datal, it does not have rigorous theoretical foundation 11 . The dependence of the TMR magnitude on the parameters of the tunnel barrier and the metal/insulator interface was taken into account in the subsequent theories. Slonczewsk 12 considered a quantum mechanical problem of tunneling of a free electron through a simple spatially uniform barrier and showed that the TMR ratio depends on the height of the potential barrier and on the effective mass of the tunneling electron inside the insulator. Later on, the influence of spin-flip scattering at the interfaces on tem- 
perature and bias-voltage dependences of the TMR was investigated by Zhang et al. 13 . It was shown that mixing of spin-up and spin-down tunnel channels leads to a decrease of the TMR.

Due to a permanent progress in the development of different methods of ab-inito calculations of the electronic properties of solids, in the last four years the transport properties of tunnel junctions were investigated using the realistic band structure of the ferromagnetic layers and the insulator (Refs. [14-20]). The systems where the conductance was calculated were ideal epitaxial $\mathrm{Fe} / \mathrm{ZnSe} / \mathrm{Fe}(001) 18$ and $\mathrm{Fe} / \mathrm{MgO} / \mathrm{Fe}(001)$ tunnel junctions 190 . For these structures the electron transport is assumed to be ballistic, i.e. the electron momentum $k_{\|}$parallel to the ferromagnet/insulator interface is conserved. Experimentally it was also demonstrated recently by Heinrich et al. 21 that $\mathrm{Fe} / \mathrm{MgO} / \mathrm{Fe}(001)$ junctions can be indeed grown by depositing the $\mathrm{MgO}$ epitaxially onto a $\mathrm{Fe}$ whisker and then depositing another Fe electrode epitaxially on a top of the $\mathrm{MgO}$. The results of ab-initio calculations showed that tunneling has more complicated behavior than the predictions of the simple barrier model proposed by Slonczewski12. The main conclusions are as follows 18 . 29 . (i) Tunneling conductance depends strongly on the symmetry of the electron states in the ferromagnetic electrodes and in the insulating layer. (ii) The decay rates of evanescent states in the barrier are different for the states with different symmetry. The slowest decay rates have the evanescent states which are compatible with $s$ symmetry. The Bloch states in the metal couple more efficiently through the interface with the decaying states of the same symmetry in the barrier. Therefore, mostly free-like $s p$-electrons from the bands with $s$ character in the ferromagnet give the essential contribution to the tunneling conductance. (iii) For thin insulating layers the tunneling current in the minority channel is dominated by the interfacial resonance states that gives rise to "hot spots" for the $k_{\|}$resolved conductance in the surface Brillouin zone. The contribution from the resonance states is substantially suppressed for the thicker barriers since the Bloch states at "hot spots" have no $s$ character 19 . Moreover, as it follows from recent discussion.22, the surface resonance states are very sensitive to the details of the interface. The asymmetry of the potential barrier and the interfacial roughness considerably reduce the contribution from the surface states to a total conductance.

Nowadays the ab-initio calculations of the TMR are possible only for ballistic junctions like above mentioned $\mathrm{Fe} / \mathrm{ZnSe} / \mathrm{Fe}$ and $\mathrm{Fe} / \mathrm{MgO} / \mathrm{Fe}$ structures which are characterized by a small lattice mismatch between the metal and the insulator, and by the well-defined band structure of the oxide or the semiconductor. In realistic junctions the electron transport has a diffuse character, i.e. when the electron crosses the ferromagnet/insulator interface its $k_{\|}$momentum is not conserved. For $\mathrm{F} / \mathrm{O} / \mathrm{F}$ tunnel junctions the most successful material until now has been alumina, $\mathrm{Al}_{2} \mathrm{O}_{3}$. The $\mathrm{Al}_{2} \mathrm{O}_{3}$ tunnel barriers are usually fabricated by the natural or plasma oxidation of the $\mathrm{Al}$ layere 8 . The subsequent structural analysis, e.g. with the use of the X-ray photoelectron spectroscopy, shows that alumina is amorphous and the obtained AlO tunnel barrier deviates from the ideal $\mathrm{Al}_{2} \mathrm{O}_{3}$ structure 10 . For a uniform coverage the $\mathrm{Al}$ film thickness is usually ranged from about 7 to $18 \AA$, depending on a type of the ferromagnetic electrode $\mathrm{Q}$. There is a small range of $\mathrm{Al}$ thicknesses that yields to the best TMR ratio for a given oxidation condition. When the $\mathrm{Al}$ layer is thin, the ferromagnet surface becomes oxidized leading to the formation of $\mathrm{CoO}$ and $\mathrm{Co}_{3} \mathrm{O}_{4}$ oxides 9 or to the $\mathrm{Fe}_{3} \mathrm{O}_{4}$ oxide 2 . On the other hand, with too thick $\mathrm{Al}$ film, an excess of $\mathrm{Al}$ metal is left unoxidized. The amorphous barriers, the roughness of the interface and its structural inhomogeneity make the rigorous ab-initio calculations of the TMR virtually impossible and therefore more simplified models are required to treat the diffuse electron transport in MTJ's.

In our previous paper23, we attempted to investigate the influence of scattering processes of the electrons (with and without spin-flip) at the interfaces on the TMR using a simple two-band (spin-up and spin-down) free-electron model. It was shown that for this simplified model spinconserving scattering may or may not lead to a decrease of the TMR depending on the amplitude of the scattering potential. In this paper, we proceed to study the diffuse electron transport in magnetic tunnel junctions of the from $\mathrm{F} / \mathrm{O} / \mathrm{F}$ with $\mathrm{F}$ 's being $3 d$ transition metal electrodes and $\mathrm{O}$ being the insulating barrier $\left(\mathrm{Al}_{2} \mathrm{O}_{3}\right)$. We use the results of ab-initio calculations to model the band structure of ferromagnetic electrodes, namely, the most important feature of these calculations that at least two groups of electrons form the total band structure: the almost free-like spin-up and spin-down bands and the narrow strongly exchange split bands. We will call the first group $s$ and the second group $d$ electrons and will consider that a periodic part of the $s$ - $d$ hybridization between bands is taken into account that results in the nonequivalence of spin-up and spin-down $s$-bands. The parameters of the adopted model can be adjusted to reproduce a value of the TMR observed in the experiments. We assume that for the case of $\mathrm{Al}_{2} \mathrm{O}_{3}$ barrier the exchange split $s$-like quasi-free electrons give the main contribution to the TMR effect. In the framework of this simplified model we will be able to investigate in a proper way the influence of electron scattering at the interfaces on the tunneling conductance.

To describe the nonideal tunnel junction we assume that defects and impurity centers (the $\mathrm{Al}$ or $\mathrm{O}$ ions, or other artificially embedded ions) are randomly distributed within few monolayers near the $\mathrm{F} / \mathrm{O}$ interface. Within the interface, an electron undergoes scattering when it comes to the defect or impurity center. We take into account these processes assuming that the hybridization between $s$ and $d$ bands changes randomly on the interface because of this parameter is the most important in the adopted model among the other ones, characterizing the scattering potential. It yields to the possibility 
of $s$-like electrons to scatter into the $d$ sub-band (and vice versa) and thus strongly affects tunneling. To treat the electron scattering on the random potential we use the coherent-potential approximation (CPA) 24 that allows to consider the case of strong scattering. We apply the generalized Kubo formalism and the Green's functions method to calculate the conductance of the system, and find the vertex corrections to the conductivity with the use of "ladder" approximation 25 combined with the CPA. It is shown that the so-called Ward identity is satisfied in the framework of this approximation that provides the necessary condition for a conservation of a tunneling current. Note, that if it is not the case, the conclusions may be completely misleading. As a result, we show that, due to substantial difference in majority and minority $d$ density of states at the Fermi energy for $3 d$ ferromagnetic metals, the inter-band $s$ - $d$ scattering on the interface can strongly reduce the TMR even down to zero value. In accordance with results of Zhang et al. 13 , it is also shown that the spin-flip scattering of electrons on the surface magnons within the interface leads to a further decrease of the TMR ratio at finite temperature.

The paper is organized as follows. In Sec. II we describe the model Hamiltonian, the calculation of the tunneling conductance and vertex corrections. The discussion of the obtained results is presented in Sec. III. Conclusions are in Sec. IV. The proof of Ward identity is given in Appendix A. The details of the derivation of the CPA equations are described in Appendix B.

\section{THEORETICAL MODEL}

\section{A. The Hamiltonian of the system}

We will consider a trilayer tunnel junction of the form $F_{1} / O / F_{3}$, where $F_{1}$ and $F_{3}$ are two semi-infinite ferromagnetic layers and $\mathrm{O}$ is a dielectric oxide spacer $\left(\mathrm{Al}_{2} \mathrm{O}_{3}\right)$. Our arguments in behalf of the two-band $s-d$ model which was briefly described in the introduction are as follows. One of the conclusions of ab-initipcalculations of the TMR for ballistic tunnel junctions 18.19 is that the expected spin-dependence of the tunneling current can be deduced from the symmetry of the Bloch states in the ferromagnet at the Fermi energy. Spin-polarized band structure for bcc Fe, fcc Ni, and fcc Co can be found in Ref. [26]. The type and symmetry of the Bloch states for different crystal faces with $\mathbf{k}_{\|}=0$ for $\mathrm{Fe}$, Co and Ni are presented in Table I (in accordance with Ref. [18]). For example, in case of $\mathrm{Fe}$ electrodes, the examination of the band structure shows that both the majority and minority bands with $s$ character in [110] and [111] directions $\left(\Lambda_{1}\right.$ and $\left.\Sigma_{1}\right)$ cross the Fermi energy. For [100] direction the band with $s$ symmetry $\left(\Delta_{1}\right)$ crosses the Fermi energy in case of the majority channel only. The similar analysis can also be performed in case of $\mathrm{Ni}$ and $\mathrm{Co}$. Thus one can assume that in the polycrystalline Fe, Co or Ni-based films the states with $s$ character present for
TABLE I: Type and symmetry of the Bloch states with $\mathbf{k}_{\|}=0$ for $\mathrm{Fe}$, Co and $\mathrm{Ni}$ for three different crystal faces (in accordance with Ref. [18]). The symmetry of these bands is as follows: $\Delta_{1}, \Sigma_{1}$, and $\Lambda_{1}(s, p, d) ; \Delta_{5}$, and $\Sigma_{2}(p$ and $d)$; and $\Delta_{2}, \Delta_{2^{\prime}}, \Sigma_{4}$, and $\Lambda_{3}(d)$.

\begin{tabular}{cccc}
\hline \hline & 100 & 110 & 111 \\
\hline $\mathrm{Fe} \uparrow$ & $\Delta_{1}, \Delta_{2^{\prime}}, \Delta_{5}$ & $\Sigma_{1}, \Sigma_{3}$ & $\Lambda_{1}$ \\
$\mathrm{Fe} \downarrow$ & $\Delta_{2}, \Delta_{2^{\prime}}, \Delta_{5}$ & $\Sigma_{1}, \Sigma_{3}$ & $\Lambda_{1}$ \\
$\mathrm{Co} \uparrow$ & $\Delta_{1}$ & $\Sigma_{1}$ & \\
$\mathrm{Co} \downarrow$ & $\Delta_{1}, \Delta_{5}$ & $\Sigma_{2}, \Sigma_{4}$ & \\
$\mathrm{Ni} \uparrow$ & $\Delta_{1}$ & $\Sigma_{1}, \Sigma_{3}$ & \\
$\mathrm{Ni} \downarrow$ & $\Delta_{1}, \Delta_{2}, \Delta_{5}$ & $\Sigma_{1}, \Sigma_{2}$ & $\Lambda_{3}$ \\
\hline \hline
\end{tabular}

both spin directions and the Bloch states will couple efficiently through the $\mathrm{F} / \mathrm{O}$ interface with $s$ states in the insulator and will decay with the equal rates in the barrier region. We will call the electrons from these bands as $s$-like electrons and will describe them as free electrons with the effective mass $m_{s} \approx m_{e}$ (where $m_{e}$ is a bare electron mass) and with different Fermi momenta $k_{s}^{F \uparrow}$ and $k_{s}^{F \downarrow}\left(k_{s}^{F \uparrow}>k_{s}^{F \downarrow}\right)$ for up and down spins. The idea about a dominant contribution of the mostly itinerant electrons to tunneling was originally proposed by M. Stearn $\$ 27$ and explained the positive polarization of the spin-dependent current in the experiments on tunneling with the superconductors 6 . According to estimations of Stearns27, in the case of Fe, $k_{s}^{F \uparrow}=1.09 \AA^{-1}$, $k_{s}^{F \downarrow}=0.42 \AA^{-1}$.

Other more localized bands (compatible with $d$ symmetry) crossing the Fermi energy also will be described by two exchange split bands with the isotropic quadratic dispersion law but with larger effective mass $m_{d} \gg m_{s}$. The Fermi momenta $k_{d}^{F \uparrow}$ and $k_{d}^{F \downarrow}$ of $d$-like electrons can be chosen to reproduce the typical for $3 d$ transition metals ratio of the values of spin-up and spin-down $d$ density of states at the Fermi energy $\varepsilon_{F}, \rho_{d}^{\uparrow}\left(\varepsilon_{F}\right): \rho_{d}^{\downarrow}\left(\varepsilon_{F}\right) \sim 1$ : 102628 In accordance with the band structure of $\mathrm{Fe}, \mathrm{Co}$ and $\mathrm{Ni26} 28$ the narrow majority and minority $d$-bands are practically filled. Therefore, the particles with a large effective mass, $m_{d}$, must be regarded as holes. The values of the Fermi momenta define the positions of band bottoms (Fig. 1) $V_{i s}^{\mu}, V_{i d}^{\mu}(\alpha=s, d ; i=1,3)$ with respect to the Fermi energy $\varepsilon_{F}$. We note, that the aim of this work is to calculate the relative change of the TMR due to scattering. Therefore the proposed model is rather adequate for this purpose since the scattering rate depends mostly on the density of states and on the matrix elements of the scattering potential.

The $\mathrm{Al}_{2} \mathrm{O}_{3}$ tunnel barriers obtained by oxidation of the $\mathrm{Al}$ film are amorphous $1 \mathrm{e}$. Concerning the $\alpha-\mathrm{Al}_{2} \mathrm{O}_{3}$ crystals, it is known from the band structure calculations 29 that the gap (which is not direct) between the upper valence band and the conduction band is of the width of $\approx 6.29 \mathrm{eV}$. The dispersion law of the lowest conduction band is not isotropic, and the effective electron masses 


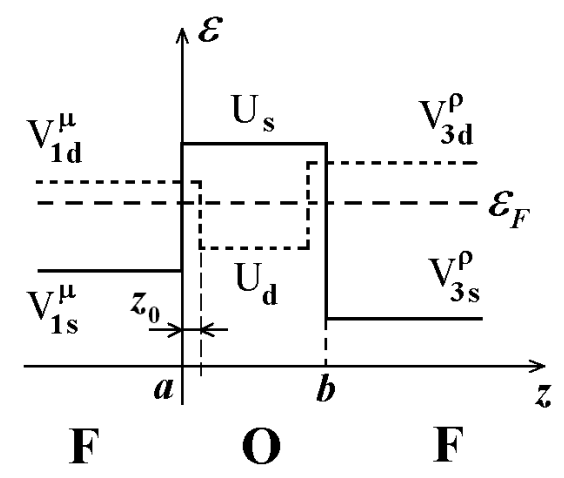

FIG. 1: The model potentials describing the propagation of an electron in the trilayer tunnel junction $\mathrm{F} / \mathrm{O} / \mathrm{F}$. The solid line corresponds to the potential profile of $s$-like electrons and the dashed line for $d$-like electrons. $V_{1(3) \alpha}^{\mu(\rho)}$ denote the spindependent band bottoms, $U_{s}$ and $U_{d}$ are the bottom and the top of the conduction and valence bands in the insulator, $\varepsilon_{F}$ is the Fermi level, $z_{0}$ is a typical width of the interface (detail description is given in the text).

along the different directions in the Brillouin zone vary from $0.16 m_{e}$ to $0.40 m_{e}$ with an average value of about $0.35 m_{e} 29$. This lowest conduction band is formed by a mixture of $\mathrm{Al} 3 s, \mathrm{O} 2 s$ and $\mathrm{O} 3 s$ orbitals. In the top of the upper valence band the dispersion curves are very flat, i.e. the effective masses of holes are large as compared with the mass of the conduction electrons. At the $\Gamma$ point, the top of the valence band consists of hybridized $\mathrm{O} 2 p$ and $\mathrm{Al} 3 p$ orbitals. In a view of that, one can describe the conduction and valence bands of the amorphous $\mathrm{Al}_{2} \mathrm{O}_{3}$ by isotropic quadratic laws with effective masses $m_{s}^{0}$ and $m_{h}^{0},\left|m_{h}^{0}\right| \gg m_{s}^{0}$.

Next, the $s$-like electrons from the ferromagnet can easily penetrate into the oxide since the lead bands with $s$ character can couple efficiently with $s$ states in the barrier, and the tunneling conductance, caused by the specular transmission of the Bloch waves through the interface, will decay for both spinup and spin-down $s$-channels with the same law as $\sim \exp \left[-2 w \sqrt{\left(2 m_{s}^{0} / \hbar^{2}\right)\left(U_{s}-\varepsilon_{F}\right)+\kappa^{2}}\right]$, where $w$ is the width of a barrier, $U_{s}$ is a bottom of the conduction band and $\kappa=k_{\|}$is the electron momentum parallel to the $\mathrm{F} / \mathrm{O}$ interface. Following MacLaren et al 18 we can suppose that $d$-like electrons from those bands in the ferromagnet without $s$ character can not couple efficiently with $s$ states in the oxide. Nevertheless, these $d$-bands have an admixture of $p$ symmetry and therefore can couple with the valence bands of the $\mathrm{Al}_{2} \mathrm{O}_{3}$. However, in the $d$ channel the tunneling conductance due to specular transmission will decay very rapidly with the thickness $w$ of the barrier, as $\sim \exp \left[-2 w \sqrt{\left(2 m_{h}^{0} / \hbar^{2}\right)\left(\varepsilon_{F}-U_{d}\right)+\kappa^{2}}\right]$, because of a large mass $m_{h}^{0}$ of holes in the alumina (here $U_{d}$ is the top of the upper valence band). The model poten- tials describing the propagation of the electron through a tunnel junction are presented in Fig. 1.

We suppose that the ferromagnet/insulator interfaces are rough and contaminated by impurity ions. The contamination of a few metal monolayers close to the $\mathrm{F} / \mathrm{O}$ interface always presents after the gxidation of the $\mathrm{Al}$ film. Other impurities (e.g. $\mathrm{Cr}, \mathrm{Ru} 30$ or $\mathrm{Cu}$ ions31) can be artificially inserted into the interface. We will characterize these structural defects by the random potentials, which may be divided into the spin-conserving and the spin-flip parts. Let $z_{1}=a$ and $z_{2}=b$ be the positions of interfaces, the $x y$-plane be the plane parallel to the interface, and the $z$-axis be the axis perpendicular to the barrier (see Fig. 1). The Hamiltonian of the system is written as:

$$
\hat{H}=\hat{H}_{0}+\hat{H}_{\text {spin-cons. }}+\hat{H}_{\text {spin-flip }}
$$

with

$$
\begin{gathered}
\hat{H}_{0}=\sum_{\mu} \int d \mathbf{r} \psi_{\mu}^{s+}(\mathbf{r})\left[-\frac{\hbar^{2}}{2 m_{s}(z)} \nabla^{2}+U_{\mu}^{s}(z)\right] \psi_{\mu}^{s}(\mathbf{r}) \\
+\sum_{\mu} \int d \mathbf{r} \psi_{\mu}^{d+}(\mathbf{r})\left[-\frac{\hbar^{2}}{2 m_{d}(z)} \nabla^{2}+U_{\mu}^{d}(z)\right] \psi_{\mu}^{d}(\mathbf{r}), \\
\hat{H}_{\text {spin-cons. }}=\sum_{\alpha=1,2} \sum_{\mu} \sum_{n} \int d \mathbf{r} \delta\left(z-z_{\alpha}\right) \delta\left(\rho-\rho_{n}^{\alpha}\right) \gamma_{n}^{\alpha} \\
\times\left[\psi_{\mu}^{s+}(\mathbf{r}) \psi_{\mu}^{d}(\mathbf{r})+\psi_{\mu}^{d+}(\mathbf{r}) \psi_{\mu}^{s}(\mathbf{r})\right], \\
\hat{H}_{\text {spin-flip }}=\sum_{\alpha=1,2} \sum_{n} \int d \mathbf{r} \delta\left(z-z_{\alpha}\right) \delta\left(\rho-\rho_{n}^{\alpha}\right) J_{n}^{\alpha} \\
\times\left[\psi_{\uparrow}^{s+}(\mathbf{r}) \psi_{\downarrow}^{s}(\mathbf{r}) \hat{S}_{-}\left(\rho_{n}^{\alpha}\right)+\psi_{\downarrow}^{s+}(\mathbf{r}) \psi_{\uparrow}^{s}(\mathbf{r}) \hat{S}_{+}\left(\rho_{n}^{\alpha}\right)\right] .
\end{gathered}
$$

Here $\mu=\uparrow, \downarrow$ is a spin suffix; $\psi_{\mu}^{s+}(\mathbf{r}), \psi_{\mu}^{d+}(\mathbf{r})$ and $\psi_{\mu}^{s}(\mathbf{r})$, $\psi_{\mu}^{d}(\mathbf{r})$ are field operators of the creation and annihilation of $s$ and $d$-type electrons with the spin $\mu$ at the point $\mathbf{r}$; $m_{s}(z)$ and $m_{d}(z)$ are the effective masses of electrons and holes in the ferromagnetic layers $\left(m_{s}\right.$ and $\left.m_{d}\right)$ or in the barrier $\left(m_{s}^{0}\right.$ and $\left.m_{h}^{0}\right)$. $U_{\mu}^{s}(z), U_{\mu}^{d}(z)$ are the spindependent step-like potentials shown in Fig. 1:

$$
\begin{gathered}
U_{\mu}^{s}(z)= \begin{cases}V_{1 s}^{\mu} & z<a \\
U_{s} & a<z<b \\
V_{3 s}^{\mu} & z>b ;\end{cases} \\
U_{\mu}^{d}(z)= \begin{cases}V_{1 d}^{\mu} & z<a+z_{0} \\
U_{d} & a+z_{0}<z<b-z_{0} \\
V_{3 d}^{\mu} & z>b-z_{0},\end{cases}
\end{gathered}
$$

where $V_{i s}^{\mu}, V_{i d}^{\mu}(i=1,3)$ are the majority $(\uparrow)$ and minority $(\downarrow)$ band bottoms in the ferromagnet, $U_{s}$ and $U_{d}$ are the bottom of the conduction band and the top of 
the upper valence band in the insulator. The positions of "steps" for the potential $U_{\mu}^{d}(z)$ are shifted by a value $z_{0}$ with respect to the points $z=a$ and $z=b$ in order to describe the finite thickness $\left(\sim z_{0}\right)$ of the interface. The explanation, why it is done in this way, is given below in the text.

$\hat{H}_{\text {spin-cons. }}$ is the spin-conserving part of the Hamiltonian, $\alpha=1,2$ are the interface numbers. To describe the defect structure of the nonideal $\mathrm{F} / \mathrm{O}$ interface we consider that the impurity ions and the ions of a ferromagnetic metal constitute the more or less random alloy of a type $\mathrm{A}_{x} \mathrm{~B}_{1-x}$ where $\mathrm{A}$ denotes the ions of the ferromagnet (Fe, $\mathrm{Co}$ or $\mathrm{Ni}$ ) and $\mathrm{B}$ denotes the impurities. Therefore, we suppose that each site $\rho_{n}^{\alpha}$ on the interface $\alpha$ is characterized by the random parameter $\gamma_{n}^{\alpha}$ of $s$ - $d$ hybridization taking two different values $\gamma_{A}$ and $\gamma_{B}$ with the probabilities $x$ and $(1-x)$, respectively. We also suppose that a periodic part of $s$ - $d$ hybridization is taken into account leading to the nonequivalence of majority and minority $s$-bands in the ferromagnet.

For the simple two-band tight-binding model of the binary alloy 35 one assumes that: 1) parameters $\varepsilon_{s}$ and $\varepsilon_{d}$ describing the positions of $s$ and $d$ bands on the energy scale are different for the alloy's components; 2) also the parameter $\gamma^{A(B)}$ of the hybridization between $s$ and $d$ bands depends on the type of an ion (A or B). In our particular case, one can not take into account the former effect since the well-defined two types of the electrons will as before exist in the vicinity of the interface and the adopted model of tunneling will not change significantly. However, the random hybridization makes possible the processes of scattering of quasi-free $s$-electrons into the localized $d$ sub-band and vice versa and, therefore, can strongly influence on tunneling.

$\hat{H}_{\text {spin-flip }}$ is a part of the Hamiltonian describing the spin-flip scattering. We take into account only the spinflip processes for $s$-like electrons since these electrons are itinerant and give the most essential contribution to the tunneling current. Operators $\hat{S}^{+}\left(\rho_{n}^{\alpha}\right), \hat{S}^{-}\left(\rho_{n}^{\alpha}\right)$ are defined as

$$
\begin{aligned}
& \hat{S}_{+}\left(\rho_{n}^{\alpha}\right)=\frac{1}{\sqrt{2 S N}} \sum_{\mathbf{q}} e^{i q \rho_{n}^{\alpha}}\left[b_{\mathbf{q}}+\ldots\right], \\
& \hat{S}_{-}\left(\rho_{n}^{\alpha}\right)=\frac{1}{\sqrt{2 S N}} \sum_{\mathbf{q}} e^{i q \rho_{n}^{\alpha}}\left[b_{\mathbf{q}}^{+}+\ldots\right] .
\end{aligned}
$$

Here $b_{\mathbf{q}}^{+}$and $b_{\mathbf{q}}$ denote the creation and annihilation operators of the surface magnons, $N$ is a number of the lattice sites on the interface, $S$ is a spin value. We used the well-known representation of the spin operators in terms of $b_{\mathbf{q}}$ and $b_{\mathbf{q}}^{+}$and left the first terms of the series. $J_{n}^{\alpha}$ is a random exchange integral which also takes the values $J_{A}$ and $J_{B}$ with probabilities $x$ and $(1-x)$.

Let us now turn back to the step-like potential for $d$ holes. As it will be clear from the following consideration, the amplitude of the effective scattering potential on the interface $(z=a)$ for $s$-like electrons (i.e. $-\operatorname{Im} \Sigma^{s s}(a)$, where $\Sigma^{s s}$ is the self-energy) is determined by the value
$\sim-x(1-x)\left(\gamma_{A}-\gamma_{B}\right)^{2} \operatorname{Im} G^{d d}(a)$, here $\operatorname{Im} G^{d d}(a)$ is the imaginary part of the $d$-electron Green's function at the point $z=a$ (density of states). If one put $z_{0}=0$ in Eq. (2), then the vertex contribution to the conductivity (that is the contribution due to tunneling assisted by interfacial scattering) will be rather small as compared with the contribution due to direct tunneling. This result is not enough accurate and is the sequence of a continual type of the model when one neglects the existence of the atomic lattice and for this reason the self-energy has a $\delta$-like behavior on the interface. However, the width $z_{0}$ of the interfacial layer is about the distance between atomic planes or even larger. For the case of bcc lattice, $z_{0}=a_{0} / 2$ for [100] direction (here $a_{0}$ is a lattice constant). One can show that for the present model with $z_{0}=0$ the imaginary part $-\operatorname{Im} G_{0}^{d d}\left(z=z^{\prime}\right)$ of the unperturbed $d$-electron Green's function (with $\Sigma^{d d}(a)=0$ ), i.e. density of states of $d$-electrons (holes), has small value at the point of interface $z=a$ and increases inside the F-layer up to the distance of the order of $z_{0} \sim a_{0}$, and then it oscillates near the average value which is approximately ten times larger than $-\operatorname{Im} G_{0}^{d d}(a)$. The period of oscillations is determined by $k_{d}^{F \uparrow}$ or $k_{d}^{F \downarrow}$, depending on the electron spin and on the orientation of magnetization in the F-layer. Such a behavior is easily understood, if to take into account that $d$-electrons (holes) are almost completely reflected on the metal/insulator interface. Thus, if one shifts the positions of "steps" for $U_{\mu}^{d}(z)$ with respect to $U_{\mu}^{s}(z)$ as it is given by (2), one can expect the more effective mechanism of scattering due to the larger value of $-\operatorname{Im} G^{d d}$, and therefore the model becomes more realistic.

\section{B. The calculation of the tunneling conductance}

To calculate the non-local conductivity we apply the Kubo formula of the linear response 32 (it is valid under the small applied voltage which is much less than the value of the energy gap in the insulator):

$$
\begin{aligned}
\sigma_{\mu \rho}\left(\mathbf{r}, \mathbf{r}^{\prime}\right)= & \frac{e^{2}}{4 \pi \hbar} \operatorname{Sp}\left\{\left\langle G_{\mu \rho}^{R}\left(\mathbf{r}, \mathbf{r}^{\prime}\right) \hat{D}_{\mathbf{r}} \hat{D}_{\mathbf{r}^{\prime}} G_{\rho \mu}^{A}\left(\mathbf{r}^{\prime}, \mathbf{r}\right)\right\rangle\right. \\
& \left.+\left\langle G_{\mu \rho}^{A}\left(\mathbf{r}, \mathbf{r}^{\prime}\right) \hat{D}_{\mathbf{r}} \hat{D}_{\mathbf{r}^{\prime}} G_{\rho \mu}^{R}\left(\mathbf{r}^{\prime}, \mathbf{r}\right)\right\rangle\right\},
\end{aligned}
$$

where a matrix operator $\hat{D}_{\mathbf{r}}$ is defined as

$$
\hat{D}_{\mathbf{r}}=\left(\begin{array}{cc}
\frac{1}{2 m_{s}(z)} \stackrel{\leftrightarrow}{\nabla}_{\mathbf{r}} & 0 \\
0 & \frac{1}{2 m_{d}(z)}
\end{array}\right)
$$

and $\stackrel{\leftrightarrow}{\nabla} \mathbf{r}=\left(\vec{\nabla}_{\mathbf{r}}-\overleftarrow{\nabla}_{\mathbf{r}}\right)$ is the asymmetric gradient operator $G_{\mu \rho}^{R}\left(\mathbf{r}, \mathbf{r}^{\prime}\right)$ and $G_{\mu \rho}^{A}\left(\mathbf{r}, \mathbf{r}^{\prime}\right)$ are the retarded and advanced $(2 \times 2)$-matrix Green's functions (with components $s s$, $s d, d s$ and $d d), \mu, \rho=\uparrow, \downarrow$ are the spin suffixes, brackets $\langle\ldots\rangle$ denote the averaging over the configurations and magnon degrees of freedom, the trace $(\mathrm{Sp})$ goes over $s$ 
and $d$ indices of the bands. Below, for convenience, it is assumed that $\hbar=1$. We will recall about $\hbar$ in the final expressions for the conductance.

To calculate the conductivity (3) of the system one has to find the Green's function of the Hamiltonian (11), which can be found by solving the following system of differential equations in the mixed $(\kappa, z)$ representation 33 :

$$
\begin{aligned}
\sum_{\gamma=s, d}\left[\varepsilon \delta^{\alpha \gamma}-\hat{H}_{\mu}^{\alpha \gamma}(z)\right] G_{\mu \kappa}^{\gamma \beta}\left(z, z^{\prime}\right)=\delta_{\alpha \beta} \delta\left(z-z^{\prime}\right) & \\
\hat{H}_{\mu}^{\alpha \gamma}(z) & =\left\{-\frac{1}{2 m_{\alpha}} \frac{\partial^{2}}{\partial z^{2}}+\frac{\kappa^{2}}{2 m_{\alpha}}+U_{\mu}^{\alpha}(z)\right\} \delta^{\alpha \gamma} \\
& +\Sigma_{\mu}^{\alpha \gamma}(a) \delta(z-a)+\Sigma_{\mu}^{\alpha \gamma}(b) \delta(z-b)
\end{aligned}
$$

where $\kappa=k_{\|}$is a projection of the electron momentum on the $x y$-plane (parallel to the F/O interface), the Greek indices $\alpha, \beta$ and $\gamma$ assume values $s$ and $d, \mu$ denotes the electron spin. $\hat{H}_{\mu}^{\alpha \beta}(z)$ is the $(2 \times 2)$-matrix linear differential operator where $\Sigma_{\mu}^{\alpha \beta}(a)$ and $\Sigma_{\mu}^{\alpha \beta}(b)(\alpha, \beta=s, d$; $\mu=\uparrow, \downarrow)$ denote the coherent potentials for spin-up and spin-down electrons which take into account the scattering of the electron by random spin-conserving and spinflip potentials on the interfaces. They were found with the use of the coherent potential approximation (CPA)24, the details of these calculations are presented in the subsequent section IIC and in Appendix B. The operator $\hat{H}_{\mu}(z)$ represents the effective single-particle Hamiltonian of the system which, however, is non-Hermitian since coherent potentials are imaginary quantities.

In order to solve Eq. (5) for the Green's functions, we will follow the procedure described below. First, let's solve the Shrödinger equation with the Hamiltonian $\hat{H}_{\mu}(z)$ :

$$
\sum_{\beta=s, d}\left[\varepsilon \delta^{\alpha \beta}-\hat{H}_{\mu}^{\alpha \beta}(z)\right] \psi_{\beta}(z)=0 .
$$

The solutions of this equation can be easily found since the potentials $U_{\mu}^{\alpha}(z)$ have a step-like form. Let us put $\varepsilon=\varepsilon_{F}+i 0$, where $\varepsilon_{F}$ is the Fermi energy, and introduce the following notations:

$$
\begin{aligned}
& k_{1 s}^{F \mu}=\sqrt{2 m_{s}\left(\varepsilon_{F}-V_{1 s}^{\mu}\right)}, \quad k_{3 s}^{F \mu}=\sqrt{2 m_{s}\left(\varepsilon_{F}-V_{3 s}^{\mu}\right)}, \\
& k_{1 d}^{F \mu}=\sqrt{2 m_{d}\left(\varepsilon_{F}-V_{1 d}^{\mu}\right)}, \quad k_{3 d}^{F \mu}=\sqrt{2 m_{d}\left(\varepsilon_{F}-V_{3 d}^{\mu}\right)}
\end{aligned}
$$

are Fermi momenta in $\mathrm{F}_{1}$ and $\mathrm{F}_{3}$ ferromagnetic layers for electrons with the spin $\mu$, and

$$
q_{2 s}^{0}=\sqrt{2 m_{s}^{0}\left(U_{s}-\varepsilon_{F}\right)}, \quad q_{2 d}^{0}=\sqrt{2 m_{h}^{0}\left(U_{d}-\varepsilon_{F}\right)} .
$$

Let also $k_{1}^{\mu \alpha}=\sqrt{\left(k_{1 \alpha}^{F \mu}\right)^{2}-\kappa^{2}}$ and $k_{3}^{\mu \alpha}=\sqrt{\left(k_{3 \alpha}^{F \mu}\right)^{2}-\kappa^{2}}$ be the components of electron momentum with spin $\mu$ along $z$-axis in $\mathrm{F}_{1}$ and $\mathrm{F}_{3}$ layers, respectively ( $\kappa$ is the inplane component of the momentum, $\alpha$ is a band index), and let $q_{2}^{\alpha}=\sqrt{\left(q_{2 \alpha}^{0}\right)^{2}+\kappa^{2}}$ be the imaginary electron momentum in the insulating layer.

Further, for convenience, we will omit indices $\mu$ and $\kappa$ in the notation of some functions. Equation (7) has four linear-independent solutions which we denote as

$$
\psi_{i}(z)=\left(\begin{array}{c}
\psi_{i}^{s}(z) \\
\psi_{i}^{d}(z)
\end{array}\right) \quad(i=1,2)
$$

and

$$
\varphi_{i}(z)=\left(\begin{array}{c}
\varphi_{i}^{s}(z) \\
\varphi_{i}^{d}(z)
\end{array}\right) \quad(i=1,2) .
$$

We choose these independent solutions so that two functions $\psi_{1}(z)$ and $\varphi_{1}(z)$ would describe two waves corresponding to the $s$-like electrons, and functions $\psi_{2}(z)$ and $\varphi_{2}(z)$ would correspond to the $d$-like electrons. Namely, in a layer $\mathrm{F}_{1}(z<a)$ the solutions $\varphi_{i}(z)$ have the form

$$
\begin{aligned}
& \varphi_{1}(z)=\left(\begin{array}{c}
\exp \left[-i k_{1}^{\mu s} z\right] \\
0
\end{array}\right) \quad z<a, \\
& \varphi_{2}(z)=\left(\begin{array}{c}
0 \\
\exp \left[-i k_{1}^{\mu d} z\right]
\end{array}\right) \quad z<a,
\end{aligned}
$$

and in a layer $\mathrm{F}_{3}(z>b)$ the solutions $\psi_{i}(z)$ are

$$
\begin{aligned}
& \psi_{1}(z)=\left(\begin{array}{c}
\exp \left[i k_{3}^{\mu s} z\right] \\
0
\end{array}\right) \quad z>b, \\
& \psi_{2}(z)=\left(\begin{array}{c}
0 \\
\exp \left[i k_{3}^{\mu d} z\right]
\end{array}\right) \quad z>b .
\end{aligned}
$$

Since $\varepsilon=\varepsilon_{F}+i 0$, then $\operatorname{Im} k_{i}^{\mu \alpha}=+0(i=1,2 ; \alpha=$ $s, d)$. Thus these solutions satisfy the following boundary conditions:

$$
\begin{array}{llll}
\psi_{i}(z) \rightarrow 0 & \text { if } & z \rightarrow+\infty & (i=1,2) \\
\varphi_{i}(z) \rightarrow 0 & \text { if } & z \rightarrow-\infty & (i=1,2) .
\end{array}
$$

Starting from expressions (8) and (9), the solutions $\varphi_{i}(z)$, $\psi_{i}(z)$ can be easily extended in two other layers. Let us introduce the matrices

$$
\begin{aligned}
& \Phi(z)=\left(\begin{array}{ll}
\varphi_{1}^{s}(z) & \varphi_{2}^{s}(z) \\
\varphi_{1}^{d}(z) & \varphi_{2}^{d}(z)
\end{array}\right), \\
& \Psi(z)=\left(\begin{array}{cc}
\psi_{1}^{s}(z) & \psi_{2}^{s}(z) \\
\psi_{1}^{d}(z) & \psi_{2}^{d}(z)
\end{array}\right) .
\end{aligned}
$$


The Wronskian of the system (7) is

$$
\Delta=\Phi^{T}(z) \hat{D}_{z} \Psi(z) .
$$

where the matrix operator $\hat{D}_{z}$ is defined similar to Eq. (何), and the subscript $T$ denotes the transposition operation. It is known from the theory of differential equations that the matrix $\Delta$ is a constant since it satisfies the equation $\frac{\partial \Delta(z)}{\partial z}=0$. Taking into account that $\varepsilon=\varepsilon_{F}+i 0$ and $\operatorname{Im} \Sigma_{\mu}^{\alpha \beta}<0$, the solution of Eq. (5) for the retarded Green's function can be written in the matrix form as

$$
\begin{aligned}
& G^{R}\left(z, z^{\prime}\right)=\Phi(z)\left[\Delta^{T}\right]^{-1} \Psi^{T}\left(z^{\prime}\right), \quad \text { if } \quad z<z^{\prime} \\
& G^{R}\left(z, z^{\prime}\right)=\Psi(z) \Delta^{-1} \Phi^{T}\left(z^{\prime}\right), \quad \text { if } \quad z>z^{\prime}
\end{aligned}
$$

To find the advanced Green's function, one has to put $\varepsilon=\varepsilon_{F}-i 0$ in Eq. (5) and assume that $\operatorname{Im} \Sigma_{\mu}^{\alpha \beta}>0$. Then we obtain

$$
G^{A}\left(z, z^{\prime}\right)=\left[G^{R}\left(z, z^{\prime}\right)\right]^{*} .
$$

In the expressions (11) and (12) the Green's functions depend on the in-plane momentum $\kappa$ and on the spin $\mu$ of the electron because solutions $\psi_{i}(z)$ and $\varphi_{i}(z)$ also depend on $\mu$ and $\kappa$.

Next, one has to find the two-point conductivity (3) of the system using the Green's functions (11) and (12). For that, we introduce the current matrices $j_{\mu}^{\psi}$ and $j_{\mu}^{\varphi}$ $(\mu=\uparrow, \downarrow)$ constructed with the use of solutions $\psi_{i}(z)$ and $\varphi_{i}(z)$, respectively:

$$
\begin{aligned}
& j^{\psi}(z)=-i \Psi^{\dagger}(z) \hat{D}_{z} \Psi(z), \\
& j^{\varphi}(z)=-i \Phi^{\dagger}(z) \hat{D}_{z} \Phi(z) .
\end{aligned}
$$

The total conductance of the system $\sigma_{\mu \rho}\left(z, z^{\prime}\right)$ may be presented in the usual form as a sum of the "bubble" part and the vertex corrections (see Fig. 2) 34 :

$$
\sigma_{\mu \rho}\left(z, z^{\prime}\right)=\delta_{\mu \rho} \sigma_{\mu}^{0}\left(z, z^{\prime}\right)+\sigma_{\mu \rho}^{\Gamma_{a}}\left(z, z^{\prime}\right)+\sigma_{\mu \rho}^{\Gamma_{b}}\left(z, z^{\prime}\right) .
$$

The "bubble" contribution to the conductance describes direct electron tunneling from the electrode to another one through the barrier when electron momentum parallel to the $\mathrm{F} / \mathrm{O}$ interface $\kappa=k_{\|}$is conserved (the specular transmission). The scattering on the interfaces affects direct tunneling so that the effective height of the potential barrier increases because the electron also has to pass through $\delta$-like potentials at the points $z=a$ and $z=b$ formed by the self-energies $\Sigma_{\mu}(a)$ and $\Sigma_{\mu}(b)$. The vertex corrections to the conductance describe the tunneling assisted by interfacial roughness - that is the processes when the electron with momentum $\kappa$ comes to the impurity center on the interface, undergoes scattering $\left(\kappa \rightarrow \kappa^{\prime}\right)$ in another channel with $\kappa^{\prime} \neq \kappa$, and then goes away to the electrode or to the barrier.

It also can be shown that the contribution to the tunneling conductance is negligibly small $\left(\sim e^{-4 q_{2}^{s}(b-a)}\right)$ from the diagram containing both vertex parts $\Gamma_{a}$ and $\Gamma_{b}$
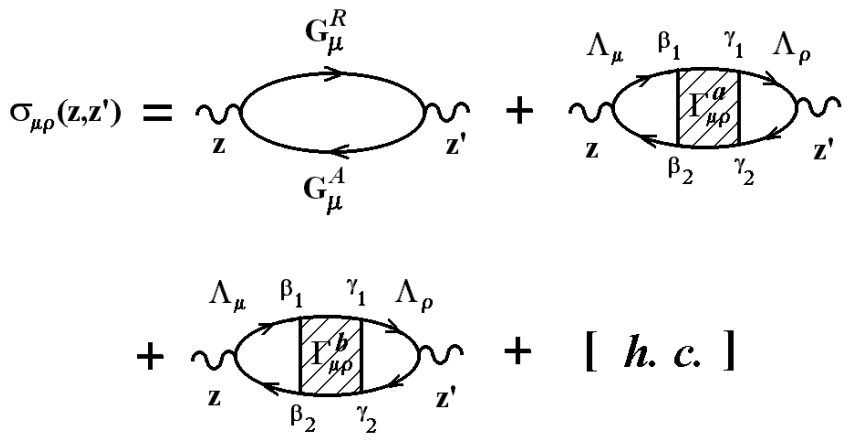

FIG. 2: The diagrammatic representation of the total twopoint nonlocal conductivity $\sigma_{\mu \rho}\left(z, z^{\prime}\right)$ as a sum of "bubble" and "vertex" parts. Here the full lines correspond to the Green's functions $G^{R}\left(z, z^{\prime}\right)$ and $G^{A}\left(z, z^{\prime}\right)$, and wavy lines denote the asymmetric gradient operator $\stackrel{\leftrightarrow}{\nabla}_{z}$ of velocity at the points $z$ and $z^{\prime}$. The shaded square designates the vertex part $\Gamma^{\mu \rho}$ at the interface; h.c. denotes the complex conjugate terms.

compared with other contributions that are of the order of $\sim e^{-2 q_{2}^{s}(b-a)}$. The diagram with vertices $\Gamma_{a}$ and $\Gamma_{b}$ corresponds to the interference of waves scattered from both interfaces. Therefore, we can neglect this interference term.

Substituting obtained expressions (11), (12) for the Green's functions and using the definition (13) for the current matrices, we come to the following results. The analytical expression for the "bubble" conductance is given by

$$
\sigma_{\mu}^{0}\left(z, z^{\prime}\right)=-\frac{e^{2}}{2 \pi \hbar A} \sum_{\kappa} \operatorname{Sp}\left\{\frac{1}{\Delta_{\mu}}\left[j_{\mu}^{\varphi}(z)\right]^{T} \frac{1}{\Delta_{\mu}^{\dagger}} j_{\mu}^{\psi}\left(z^{\prime}\right)\right\},
$$

here it is assumed that $z<z^{\prime}, \Delta_{\mu}$ is the Wronskian (10), $A$ denotes the junction area.

For $z<a, z^{\prime}>b$, the vertex corrections from the left and from the right interfaces can be written as

$$
\begin{aligned}
\sigma_{\mu \rho}^{\Gamma_{a}}\left(z, z^{\prime}\right)= & -\frac{e^{2}}{2 \pi \hbar A^{2}} \sum_{\kappa \kappa^{\prime}} \operatorname{Sp}\left\{\Lambda_{\mu \kappa}^{\beta_{1} \beta_{2}}(z, a)\right. \\
& \left.\times \Gamma_{a}^{\mu \rho}\left(\begin{array}{cc}
\beta_{1} & \gamma_{1} \\
\beta_{2} & \gamma_{2}
\end{array}\right) \Lambda_{\rho \kappa^{\prime}}^{\gamma_{1} \gamma_{2}}\left(a, z^{\prime}\right)\right\} \\
\sigma_{\mu \rho}^{\Gamma_{b}}\left(z, z^{\prime}\right)= & -\frac{e^{2}}{2 \pi \hbar A^{2}} \sum_{\kappa \kappa^{\prime}} \operatorname{Sp}\left\{\Lambda_{\mu \kappa}^{\beta_{1} \beta_{2}}(z, b)\right. \\
& \left.\times \Gamma_{b}^{\mu \rho}\left(\begin{array}{cc}
\beta_{1} & \gamma_{1} \\
\beta_{2} & \gamma_{2}
\end{array}\right) \Lambda_{\rho \kappa^{\prime}}^{\gamma_{1} \gamma_{2}}\left(b, z^{\prime}\right)\right\}
\end{aligned}
$$

where summation is also performed over repeating indices $\beta_{i}, \gamma_{i}=s, d$. Here $\Gamma_{a}^{\mu \rho}\left(\begin{array}{ll}\beta_{1} & \gamma_{1} \\ \beta_{2} & \gamma_{2}\end{array}\right)$ and $\Gamma_{b}^{\mu \rho}\left(\begin{array}{ll}\beta_{1} & \gamma_{1} \\ \beta_{2} & \gamma_{2}\end{array}\right)$ are the vertex parts on the interfaces $a$ and $b . \Lambda_{\mu \kappa}$ are $(2 \times 2)-$ 
matrices, their components are defined by

$$
\begin{gathered}
\Lambda_{\mu \kappa}^{\beta_{1} \beta_{2}}(z, a)=\operatorname{Sp}\left\{\frac{1}{\Delta_{\mu}} j_{\mu}^{\varphi T}(z) \frac{1}{\Delta_{\mu}^{\dagger}}\left[\rho_{\mu}^{\psi}(a)\right]^{\beta_{1} \beta_{2}}\right\}, \\
\Lambda_{\mu \kappa}^{\gamma_{1} \gamma_{2}}\left(a, z^{\prime}\right)=\operatorname{Sp}\left\{\frac{1}{\Delta_{\mu}}\left[\rho_{\mu}^{\varphi T}(a)\right]^{\gamma_{1} \gamma_{2}} \frac{1}{\Delta_{\mu}^{\dagger}} j^{\psi}\left(z^{\prime}\right)\right\},
\end{gathered}
$$

where $\left[\rho_{\mu}^{\psi}(a)\right]^{\beta_{1} \beta_{2}}$ and $\left[\rho_{\mu}^{\varphi}(a)\right]^{\gamma_{1} \gamma_{2}}$ are density matrices with the components:

$$
\begin{aligned}
& {\left[\rho_{\mu}^{\psi}(a)\right]_{i k}^{\beta_{1} \beta_{2}}=\psi_{i}^{\beta_{1} *}(a) \psi_{k}^{\beta_{2}}(a) \quad(i, k=s, d),} \\
& {\left[\rho_{\mu}^{\varphi}(a)\right]_{i k}^{\gamma_{1} \gamma_{2}}=\varphi_{i}^{\gamma_{1} *}(a) \varphi_{k}^{\gamma_{2}}(a) \quad(i, k=s, d) .}
\end{aligned}
$$

The expressions similar to expressions (17) may be also written for the matrices $\Lambda_{\mu \kappa}(z, b), \Lambda_{\rho \kappa}\left(b, z^{\prime}\right)$ and for other position of the points $z, z^{\prime}$ with respect to $a$ and $b$. The vertex parts $\Gamma^{\mu \rho}$ and $\Gamma_{b}^{\mu \rho}$ are calculated in the "ladder" approximation 25 . The derivation of the equation for $\Gamma^{\mu \rho}$ is presented below in the subsection IID.

We have to note, that coherent potentials $\Sigma_{\mu}(a), \Sigma_{\mu}(b)$ calculated in the framework of the CPA and vertices $\Gamma_{a}^{\mu \rho}$, $\Gamma_{b}^{\mu \rho}$ calculated in the "ladder" approximation satisfy the so-called Ward identity which in our case, for example, for the interface $z=a$ can be written as follows (for the details, see Appendix A):

$$
\operatorname{Im} \Sigma_{\mu}^{\beta_{1} \beta_{2}}(a)=\sum_{\rho=\uparrow, \downarrow} \Gamma_{a}^{\mu \rho}\left(\begin{array}{ll}
\beta_{1} & \gamma_{1} \\
\beta_{2} & \gamma_{2}
\end{array}\right) \frac{1}{A} \sum_{\kappa}\left\{\operatorname{Im} G_{\rho \kappa}^{\gamma_{1} \gamma_{2}}(a)\right.
$$

$$
\left.-G_{\rho \kappa}^{\gamma_{1} \alpha_{1} *}(a) G_{\rho \kappa}^{\gamma_{2} \alpha_{2}}(a) \operatorname{Im} \Sigma_{\rho \kappa}^{\alpha_{1} \alpha_{2}}(a)\right\} .
$$

Here the summation is also performed over repeating indices $\gamma_{i}$ and $\alpha_{i}$. The fulfillment of (19) provides the necessary condition of the nondivergence of the current through the tunnel junction:

$$
\frac{\partial}{\partial z^{\prime}} \sigma_{\mu}^{0}\left(z, z^{\prime}\right)+\frac{\partial}{\partial z^{\prime}} \sum_{\rho=\uparrow, \downarrow}\left[\sigma_{\mu \rho}^{\Gamma_{a}}\left(z, z^{\prime}\right)+\sigma_{\mu \rho}^{\Gamma_{b}}\left(z, z^{\prime}\right)\right]=0 .
$$

According to Eq. (20), the total conductance of the system is a constant value. In view of that, we will derive the exact expression for $\sigma_{\mu \rho}\left(z, z^{\prime}\right)$ evaluating the conductance at points $z=a-0$ and $z^{\prime}=b+0$, i.e. at the left and at the right sides from the interface. The Wronskian matrix $\Delta_{\mu}$ and the current matrices $j_{\mu}^{\varphi}(z)$ and $j_{\mu}^{\psi}\left(z^{\prime}\right)$ are expressed in terms of matrices $\Phi(z)$ and $\Psi(z)$ - these matrices, as follows from Eq. (11), determine the Green's function. The straightforward evaluation of the conductance according to formulae (15 17) leads to the result that $\sigma_{\mu \rho}(a, b)$ is expressed in terms of the retarded Green's functions at the points of interfaces $G_{\mu}(a)=G_{\mu}^{R}\left(z=z^{\prime}=a\right)$ and $G_{\mu}(b)=G_{\mu}^{R}\left(z=z^{\prime}=b\right)$ constructed according to Eq. (11). The explicit form of $G_{\mu}(a)$ in the $(\kappa, z)$ representation is given by the expression:

$$
G_{\mu}(a)=\left(\begin{array}{c}
\frac{k_{1}^{\mu s}}{2 m_{s}}\left(i-\operatorname{cotan} \varphi_{1}^{\mu s}\right)-\Sigma_{\mu}^{s s}(a) \\
-\Sigma_{\mu}^{d s}(a)
\end{array}\right.
$$

where

$$
\tan \varphi_{1}^{\mu s}=\frac{k_{1}^{\mu s}}{q_{2}^{s}} \frac{m_{s}^{0}}{m_{s}}, \quad \tan \varphi_{1}^{\mu d}=\frac{k_{1}^{\mu d}}{q_{2}^{d}} \frac{m_{h}^{0}}{m_{d}} .
$$

The similar expression can be written for the Green's function $G_{\mu}(b)$ on the right interface. For that, one has to make the substitutions $k_{1}^{\mu \alpha} \rightarrow k_{3}^{\mu \alpha}, \Sigma_{\mu}^{\alpha \beta}(a) \rightarrow \Sigma_{\mu}^{\alpha \beta}(b)$, $\varphi_{1}^{\mu \alpha} \rightarrow \varphi_{3}^{\mu \alpha}$ in the expression for $G_{\mu}(a)$. Here $m_{\alpha}(\alpha=$ $s, d)$ and $m_{s(h)}^{0}$ are the effective electron (hole) masses in the ferromagnetic and insulating layers; $k_{i}^{\mu \alpha}$ and $q_{2}^{\alpha}(i=$ $1,3, \alpha=s, d)$ are the functions on $\kappa$ introduced above in the text after Eq. (]). Let us define the "transport" density of states as follows:

$$
\begin{aligned}
& A_{\mu}(a)=G_{\mu}^{\dagger}(a) \tilde{j}_{\mu}^{\varphi} G_{\mu}(a), \\
& A_{\mu}(b)=G_{\mu}^{\dagger}(b) \tilde{j}_{\mu}^{\psi} G_{\mu}(b),
\end{aligned}
$$

where

$$
\tilde{j}_{\mu}^{\varphi}=\left(\begin{array}{cc}
\frac{k_{1}^{\mu s}}{m_{s}} & 0 \\
0 & \frac{k_{1}^{\mu d}}{m_{d}}
\end{array}\right), \quad \tilde{j}_{\mu}^{\psi}=\left(\begin{array}{cc}
\frac{k_{3}^{\mu s}}{m_{s}} & 0 \\
0 & \frac{k_{3}^{\mu d}}{m_{d}}
\end{array}\right) .
$$

Expression (15) for the "bubble" conductance then reads

$$
\begin{aligned}
\sigma_{\mu}^{0}(a, b)= & \frac{e^{2}}{2 \pi \hbar A} \sum_{\kappa} \operatorname{Sp}\left\{\lambda_{b}^{-1}\left(\frac{\hat{q}}{m}\right) \lambda_{a}^{-1}\right. \\
& \left.\times A_{\mu}^{T}(a) \lambda_{a}^{-1}\left(\frac{\hat{q}}{m}\right) \lambda_{b}^{-1} A_{\mu}(b)\right\},
\end{aligned}
$$

where

$$
\lambda_{a}=\left(\begin{array}{cc}
e^{q_{2}^{s} z_{0}} & 0 \\
0 & \lambda_{a}^{d}
\end{array}\right), \quad \lambda_{a}^{d}=\frac{\sin \left(k_{1}^{\mu d} z_{0}+\varphi_{1}^{\mu d}\right)}{\sin \varphi_{1}^{\mu d}},
$$




$$
\begin{gathered}
\lambda_{b}=\left(\begin{array}{cc}
e^{q_{2}^{s} z_{0}} & 0 \\
0 & \lambda_{b}^{d}
\end{array}\right), \quad \lambda_{b}^{d}=\frac{\sin \left(k_{3}^{\mu d} z_{0}+\varphi_{3}^{\mu d}\right)}{\sin \varphi_{3}^{\mu d}}, \\
\left(\frac{\hat{q}}{m}\right)=\left(\begin{array}{cc}
\frac{q_{2}^{s}}{m_{s}^{0}} e^{-q_{2}^{s} \tilde{w}} & 0 \\
0 & \frac{q_{2}^{d}}{m_{h}^{0}} e^{-q_{2}^{d} \tilde{w}}
\end{array}\right),
\end{gathered}
$$

and $\tilde{w}=b-a-2 z_{0}$ is the "width" of $d$-barrier.

For the vertex correction from the left interface $(z=a)$ we obtain

$$
\begin{aligned}
\sigma_{\mu \rho}^{\Gamma_{a}}(a, b)= & -\frac{e^{2}}{2 \pi \hbar A^{2}} \sum_{\kappa \kappa^{\prime}} \Lambda_{\mu \kappa}^{\beta_{1} \beta_{2}}(a, a) \\
& \times \Gamma_{a}^{\mu \rho}\left(\begin{array}{ll}
\beta_{1} & \gamma_{1} \\
\beta_{2} & \gamma_{2}
\end{array}\right) \Lambda_{\rho \kappa^{\prime}}^{\gamma_{1} \gamma_{2}}(a, b) \\
\Lambda_{\mu}(a, a)= & -A_{\mu}(a), \\
\Lambda_{\rho}(a, b)= & G_{\rho}^{\dagger}(a) \lambda_{a}^{-1}\left(\frac{\hat{q}}{m}\right) \lambda_{b}^{-1} A_{\rho}(b) \\
& \times \lambda_{b}^{-1}\left(\frac{\hat{q}}{m}\right) \lambda_{a}^{-1} G_{\rho}(a) .
\end{aligned}
$$

In the similar way, the vertex correction from the right interface $(z=b)$ reads

$$
\begin{aligned}
\sigma_{\mu \rho}^{\Gamma_{b}}(a, b)= & -\frac{e^{2}}{2 \pi \hbar A^{2}} \sum_{\kappa \kappa^{\prime}} \Lambda_{\mu \kappa}^{\beta_{1} \beta_{2}}(a, b) \\
& \times \Gamma_{b}^{\mu \rho}\left(\begin{array}{cc}
\beta_{1} & \gamma_{1} \\
\beta_{2} & \gamma_{2}
\end{array}\right) \Lambda_{\rho \kappa^{\prime}}^{\gamma_{1} \gamma_{2}}(b, b), \\
\Lambda_{\mu}(a, b)= & G_{\mu}^{\dagger}(b) \lambda_{b}^{-1}\left(\frac{\hat{q}}{m}\right) \lambda_{a}^{-1} A_{\mu}(a) \\
& \times \lambda_{a}^{-1}\left(\frac{\hat{q}}{m}\right) \lambda_{b}^{-1} G_{\mu}(b), \\
\Lambda_{\rho}(b, b)= & -A_{\rho}(b) .
\end{aligned}
$$

Expressions (23 25) determine the total conductance of the system.

\section{The CPA equations}

The scattering of the electron by random potentials within the interface (terms $\hat{H}_{\text {spin-cons. }}$ and $\hat{H}_{\text {spin-flip }}$ in the Hamiltonian) is taken into account by introducing the self-energy operators $\Sigma_{\mu}^{\alpha \beta}(a)$ and $\Sigma_{\mu}^{\alpha \beta}(b)$ into the effective single-particle Hamiltionian (6). To calculate the selfenergies apply the coherent-potential approximation (CPA)24,B5. Let's denote kets $\left|\gamma, \mu, \rho_{n}^{\alpha}\right\rangle=|\gamma, \mu\rangle \otimes\left|\rho_{n}^{\alpha}\right\rangle$ corresponding to the Wannier states of the electron on the interface $\alpha(\alpha=1,2)$ at the given site $\rho_{n}^{\alpha}$ in the $(x, y)$-plane, where $\gamma$ refers to the state $s$ or $d$ and $\mu$ is the spin. The symbol $\otimes$ denotes the direct product. The problem of finding of the single-particle Green's function $G_{\mu \rho}\left(\mathbf{r}, \mathbf{r}^{\prime}\right)$ of the many-body Hamiltonian (11) is reduced to the related single electron problem of the propagation of the electron in a random interfacial potential

$$
\begin{aligned}
\hat{V} & =\sum_{n ; \alpha=1,2}\left|\rho_{n}^{\alpha}\right\rangle\left(\hat{v}_{n}^{(\mathrm{el}) \alpha}+\hat{v}_{n}^{(\mathrm{sf}) \alpha}\right)\left\langle\rho_{n}^{\alpha}\right| \\
& =\sum_{n ; \alpha=1,2}\left|\rho_{n}^{\alpha}\right\rangle \hat{v}_{n}^{\alpha}\left\langle\rho_{n}^{\alpha}\right|,
\end{aligned}
$$

where $\hat{v}_{n}^{\alpha}=\hat{v}_{n}^{(\mathrm{el}) \alpha}+\hat{v}_{n}^{(\mathrm{sf}) \alpha}$ and the summation goes over the interface number $\alpha$ and over the sites $n$. Here

$$
\hat{v}_{n}^{(\mathrm{el}) \alpha}=\sum_{\mu=\uparrow, \downarrow} \gamma_{n}^{\alpha}\{|s, \mu\rangle\langle d, \mu|+| d, \mu\rangle\langle s, \mu|\}
$$

is the random potential of $s-d$ hybridization associated with the site $n$ and

$$
\hat{v}_{n}^{(\mathrm{sf}) \alpha}=J_{n}^{\alpha}\left\{|s, \uparrow\rangle \hat{S}_{-}\left(\rho_{n}^{\alpha}\right)\langle s, \downarrow|+| s, \downarrow\rangle \hat{S}_{+}\left(\rho_{n}^{\alpha}\right)\langle s, \uparrow|\right\}
$$

is the exchange-like interaction with the surface magnons. The random quantities $\gamma_{n}^{\alpha}$ and $J_{n}^{\alpha}$ used here were introduced in Sec. IIA.

Now one can formulate the CPA by the ordinary way and the only difference with respect to the usual situation of the bulk scattering is that the initial Green's functions have to be calculated for the trilayer system. We assume that the averaged propagator of the system $G_{\mu}^{\alpha \beta}\left(\mathbf{r}, \mathbf{r}^{\prime}\right)$ differs from the initial Green's function, corresponding to the Hamiltonian $\hat{H}_{0}$ [see Eq. (11)], by the self-energy correction in Eqs. (5) , (5). This means that the system behaves as if coherent potentials $\Sigma_{\mu}^{\alpha \beta}$ had been assigned to each site of the interface $a$ and $b$. After the introducing of the effective medium $\hat{\Sigma}^{\alpha}$, each site $\rho_{n}^{\alpha}$ becomes a source of the random potential $\hat{u}_{n}^{\alpha}=\hat{v}_{n}^{\alpha}-\hat{\Sigma}^{\alpha}$. The single-site $t$-matrix associated with potential $\hat{u}_{n}^{\alpha}$ is given by

$$
\hat{t}_{n}^{\alpha}=\frac{1}{1-\left\{\hat{v}_{n}^{\alpha}-\hat{\Sigma}^{\alpha}\right\} \hat{G}\left(z_{\alpha}\right)}\left\{\hat{v}_{n}^{\alpha}-\hat{\Sigma}^{\alpha}\right\},
$$

where $z_{\alpha}=a$ or $b$,

$$
\begin{aligned}
\hat{\Sigma}^{\alpha} & =\sum_{\mu=\uparrow, \downarrow} \sum_{\beta, \gamma=s, d}|\beta, \mu\rangle \Sigma_{\mu}^{\beta \gamma}\left(z_{\alpha}\right)\langle\gamma, \mu|, \\
\hat{G}\left(z_{\alpha}\right) & =\sum_{\mu=\uparrow, \downarrow} \sum_{\beta, \gamma=s, d}|\beta, \mu\rangle G_{\mu}^{\beta \gamma}\left(z_{\alpha}\right)\langle\gamma, \mu| .
\end{aligned}
$$

Here

$$
G_{\mu}^{\beta \gamma}\left(z_{\alpha}\right)=\int_{0}^{\kappa_{\max }} G_{\mu}^{\alpha \beta}\left(z_{\alpha}, \kappa\right) \frac{\kappa d \kappa}{2 \pi}
$$

is the averaged Green's function at the interface $\alpha$ which is expressed via $\hat{\Sigma}^{\alpha}$ in accordance with Eq. 211. The upper limit $\kappa_{\max }$ is a cut-off of the in-plane momentum which originates from the finite size of the Brillouin zone. For that, one has to substitute the Brillouin zone's projection onto $\left(k_{x}, k_{y}\right)$-plane by a circle of the same square with a radius $\kappa_{\max }$. For the bcc lattice, 
$\kappa_{\max }=2 \sqrt{\pi / \sqrt{2}} / a_{0}$, where $a_{0}$ is a lattice constant. The single-site $t$-matrix 28) is obviously different for different sites. At the same time it is supposed that scattering by the random potential is taken into account in the averaged propagator $\hat{G}\left(z_{\alpha}\right)$ by the self-energy operator $\hat{\Sigma}^{\alpha}$. Therefore, we require that the ensemble average of the single-site $t$-matrix vanishes, i.e.,

$$
\left\langle\hat{t}_{n}^{\alpha}\left(\hat{\Sigma}^{\alpha}\right)\right\rangle=x\left\langle\hat{t}_{A}^{\alpha}\right\rangle_{b}+y\left\langle\hat{t}_{B}^{\alpha}\right\rangle_{b}=0
$$

Here $t_{A}^{\alpha}$ and $t_{B}^{\beta}$ are the values of the single-site $t$-matrix associated with a given site $n$ which is occupied by the Atype ion (ferromagnet's ion) or by the B-type ion (impurity), respectively. Brackets $\langle\ldots\rangle_{b}$ denote the averaging over magnon degrees of freedom. The equation (31) is the well-known self-consistent coherent potential approximation (CPA)2435 that implicitly determines the self-energy operator $\hat{\Sigma}^{\alpha}$. The CPA equations (31) are formulated for the particular case of electron scattering within the $\mathrm{F} / \mathrm{O}$ interface. The straightforward calculations show that the single-site $t$-matrix $\hat{t}_{n}^{\alpha}$ can be represented in the form:

$$
\hat{t}_{n}^{\alpha}=\left(\begin{array}{cc}
\hat{t}_{n}^{\alpha \uparrow}\left(\hat{n}_{+}\right) & \hat{t}_{n}^{\alpha+}\left(\hat{n}_{-}\right) \hat{S}_{-} \\
\hat{t}_{n}^{\alpha-}\left(\hat{n}_{+}\right) \hat{S}_{+} & \hat{t}_{n}^{\alpha \downarrow}\left(\hat{n}_{-}\right)
\end{array}\right)
$$

with respect to spin-up and spin-down subspaces. Here $\hat{n}_{+}=\hat{S}_{+} \hat{S}_{-}, \hat{n}_{-}=\hat{S}_{-} \hat{S}_{+}$, and the blocks $\hat{t}_{n}^{\alpha \pm}, \hat{t}_{n}^{\alpha \uparrow(\downarrow)}$ are $(2 \times 2)$-matrices, functionally depending on $\hat{n}_{+}$and $\hat{n}_{-}$, with the components designated by indices of bands $s$ and $d$.

To satisfy the condition (31), one has to consider only the spin-conserving part of this equation as long as $\left\langle t_{n}^{\alpha \pm} \hat{S}_{\mp}\right\rangle_{b}=0$ since the expression to be averaged contains unequal number of the creation and annihilation operators of the magnons. In order to calculate $\left\langle t_{n}^{\alpha \uparrow(\downarrow)}\right\rangle_{b}$ we adopted the further approximation and assumed that

$$
\begin{aligned}
\left\langle t_{n}^{\alpha \uparrow}\left(\hat{n}_{+}\right)\right\rangle_{b} & =\hat{t}_{n}^{\alpha \uparrow}(n), \\
\left\langle t_{n}^{\alpha \downarrow}\left(\hat{n}_{-}\right)\right\rangle_{b} & =\hat{t}_{n}^{\alpha \downarrow}(n),
\end{aligned}
$$

here $n=n(T)$ is the average number of magnons at the given temperature. In other words, we substituted the operators $\hat{n}_{ \pm}$by its averaged values. The function $n(T)$ is given by the familiar expression

$$
n(T)=\int \frac{d^{2} \mathbf{q}}{(2 \pi)^{2}} \frac{1}{e^{\beta \omega(\mathbf{q})}-1}
$$

where $\omega(\mathbf{q})$ is the spectrum of surface magnons. The approximation we made to take into account the spinflip processes is the static approximation for magnons. The inelasticity of the electron-magnon processes may be taken into account in the energy conservation rule. However, the characteristic magnon energy $\hbar \omega_{0}$ is much less than the Fermi energy $\varepsilon_{F}$, and in the first approximation one can neglect this energy $\hbar \omega_{0}$ - it becomes important in the case of finite voltages when the process of emitting of a magnon by a hot electron influences on

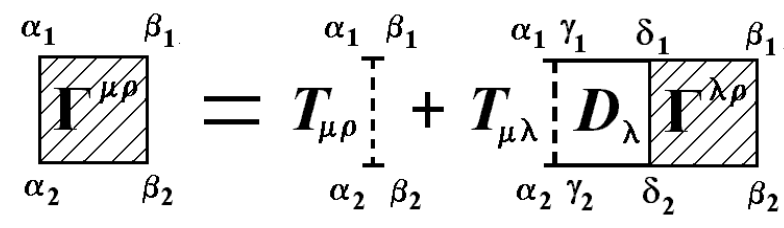

FIG. 3: The diagrammatic representation of the equation (see the text) corresponding to the calculation of the vertex part $\Gamma^{\mu \rho}$ in the "ladder" approximation.

the form of $I-V$ dependence. We, therefore, restricted our calculation to the case of small voltage bias with the voltage less than $\hbar \omega_{0}$. Within this approximation, for the system of CPA equations we get

$$
\begin{aligned}
& t_{A}^{\alpha \uparrow}(n) x+t_{B}^{\alpha \uparrow}(n) y=0, \\
& t_{A}^{\alpha \downarrow}(n) x+t_{B}^{\alpha \downarrow}(n) y=0 .
\end{aligned}
$$

As far as matrices $t_{n}^{\alpha \uparrow(\downarrow)}$ are $(2 \times 2)$-blocks, thus for the general case Eq. (34) represents the system of two matrix equations for 8 unknown quantities $\Sigma_{\mu}^{\beta \gamma}\left(z_{\alpha}\right)$. The system (34) is one of the possible forms of the CPA equations. But actually we used another representation of the CPA which was more convenient for the numerical implementation. For that we exploited the augmented-space formalism by Mookerjee 36 , and the details are presented in Appendix B.

\section{The vertex corrections}

To find the vertex corrections we used the "ladder" approximation combined with the CPA, that was originally proposed by Velický25. The diagrammatic representation of the "ladder" approximation is given in Fig. 3. Since the scattering potentials on the different interfaces (at the points $a$ and $b$ ) do not correlate with each other, the vertex parts $\Gamma_{a(b)}^{\mu \rho}\left(\begin{array}{ll}\beta_{1} & \gamma_{1} \\ \beta_{2} & \gamma_{2}\end{array}\right)$ can be found independently for each interface and we will omit interface suffix in the subsequent expressions. Let $T^{\mu \rho}\left(\begin{array}{ll}\beta_{1} & \gamma_{1} \\ \beta_{2} & \gamma_{2}\end{array}\right)=\left\langle\left(t_{n}^{\alpha}\right)_{\mu \rho}^{* \beta_{1} \gamma_{1}}\left(t_{n}^{\alpha}\right)_{\rho \mu}^{\gamma_{2} \beta_{2}}\right\rangle$ be the average of the product of two random $t$-matrices over configurations and magnon distribution, where as before $\mu, \rho$ are spin indices and $\beta, \gamma$ are orbital indices. On the diagram in Fig.3, it is presented by the dashed line. The analytical expression for the correlator $T^{\mu \rho}\left(\begin{array}{ll}\beta_{1} & \gamma_{1} \\ \beta_{2} & \gamma_{2}\end{array}\right)$ can be found in accordance with the adopted approximate scheme of averaging over the magnon degrees of freedom. Taking into account Eq. (32) we obtain:

$$
\begin{aligned}
T^{\uparrow \uparrow}\left(\begin{array}{cc}
\beta_{1} & \gamma_{1} \\
\beta_{2} & \gamma_{2}
\end{array}\right)= & x t_{A}^{* \uparrow}(n)^{\beta_{1} \gamma_{1}} t_{A}^{\uparrow}(n)^{\gamma_{2} \beta_{2}} \\
& +y t_{B}^{* \uparrow}(n)^{\beta_{1} \gamma_{1}} t_{B}^{\uparrow}(n)^{\gamma_{2} \beta_{2}}
\end{aligned}
$$




$$
\begin{aligned}
T^{\downarrow \downarrow}\left(\begin{array}{ll}
\beta_{1} & \gamma_{1} \\
\beta_{2} & \gamma_{2}
\end{array}\right)= & x t_{A}^{* \downarrow}(n)^{\beta_{1} \gamma_{1}} t_{A}^{\downarrow}(n)^{\gamma_{2} \beta_{2}} \\
& +y t_{B}^{* \downarrow}(n)^{\beta_{1} \gamma_{1}} t_{B}^{\downarrow}(n)^{\gamma_{2} \beta_{2}}, \\
T^{\uparrow \downarrow}\left(\begin{array}{ll}
\beta_{1} & \gamma_{1} \\
\beta_{2} & \gamma_{2}
\end{array}\right)= & x n t_{A}^{*+}(n)^{\beta_{1} \gamma_{1}} t_{A}^{-}(n)^{\gamma_{2} \beta_{2}} \\
& +y n t_{B}^{*+}(n)^{\beta_{1} \gamma_{1}} t_{B}^{-}(n)^{\gamma_{2} \beta_{2}}, \\
T^{\downarrow \uparrow}\left(\begin{array}{cc}
\beta_{1} & \gamma_{1} \\
\beta_{2} & \gamma_{2}
\end{array}\right)= & x n t_{A}^{*-}(n)^{\beta_{1} \gamma_{1}} t_{A}^{+}(n)^{\gamma_{2} \beta_{2}} \\
& +y n t_{B}^{*-}(n)^{\beta_{1} \gamma_{1}} t_{B}^{+}(n)^{\gamma_{2} \beta_{2}},
\end{aligned}
$$

where $t_{A(B)}^{\uparrow(\downarrow)}(n)$ and $t_{A(B)}^{ \pm}(n)$ are the components of the single-site $t$-matrix, Eq. (32). We also define the operator

$$
\begin{aligned}
D_{\mu}^{\alpha}\left(\begin{array}{cc}
\beta_{1} & \gamma_{1} \\
\beta_{2} & \gamma_{2}
\end{array}\right)= & \frac{1}{A} \sum_{\kappa}\left[G_{\kappa \mu}^{* \beta_{1} \gamma_{1}}\left(z_{\alpha}\right) G_{\kappa \mu}^{\beta_{2} \gamma_{2}}\left(z_{\alpha}\right)\right] \\
& -G_{\mu}^{* \beta_{1} \gamma_{1}}\left(z_{\alpha}\right) G_{\mu}^{\beta_{2} \gamma_{2}}\left(z_{\alpha}\right)
\end{aligned}
$$

denoting the propagator of the pair of electrons in the "ladder" equation at the interface $z_{\alpha}$. Its definition follows from the fact that in the diagram representation of the CPA the multiple scattering on the given site is assumed to be incorporated into the single-site $t$-matrix $t_{n}^{\alpha}$, corresponding to the single vertex of any diagram. Due to that, the subsequent sites in the "ladder" diagrammatic equation (Fig.3) must not reproduce each other. Therefore, the necessary correction is subtracted in Eq. (36). The summation over $\kappa$ goes up to $\kappa_{\max }$ similar to Eq. (30). After that definitions the analytical equation for the vertex part reads as

$$
\begin{aligned}
\Gamma^{\mu \rho}\left(\begin{array}{ll}
\alpha_{1} & \beta_{1} \\
\alpha_{2} & \beta_{2}
\end{array}\right)= & T_{\mu \rho}\left(\begin{array}{ll}
\alpha_{1} & \beta_{1} \\
\alpha_{2} & \beta_{2}
\end{array}\right)+T_{\mu \lambda}\left(\begin{array}{ll}
\alpha_{1} & \gamma_{1} \\
\alpha_{2} & \gamma_{2}
\end{array}\right)(37) \\
& \times D_{\lambda}\left(\begin{array}{ll}
\gamma_{1} & \delta_{1} \\
\gamma_{2} & \delta_{2}
\end{array}\right) \Gamma^{\lambda \rho}\left(\begin{array}{ll}
\delta_{1} & \beta_{1} \\
\delta_{2} & \beta_{2}
\end{array}\right)
\end{aligned}
$$

Eq. (37) is the ordinary system of linear equations. It can be proved that the vertex parts found with the use of the "ladder" approximation and self-energies found with the use of the CPA satisfy the Ward identity (19), and hence the total tunneling current is a constant value $j(z)=j_{0}$, i.e. it does not depend on $z$. The proof of the Ward identity is presented in Appendix A.

\section{RESULTS AND DISCUSSION}

We considered the case of $\mathrm{Fe} / \mathrm{Al}_{2} \mathrm{O}_{3} / \mathrm{Fe}$ tunnel junction. The following parameters were chosen to describe the system. According to estimations of Stearns 20 , for the itinerant $s$-like electrons in Fe we set $k_{s}^{F \uparrow}=1.09 \AA^{-1}$, $k_{s}^{F \downarrow}=0.42 \AA^{-1}$, and $m_{s}=1.0 m_{e}$ (here $m_{e}$ is bare electron mass). For the more localized $d$-electrons (holes), we put $m_{d}=-10.0 m_{e}$. The $d$ density of states at the Fermi energy is larger for the minority spin band 26 , therefore, $k_{d}^{F \downarrow}>k_{d}^{F \uparrow}$, and we put $k_{d}^{F \uparrow}=0.45 \AA^{-1}$,
$k_{d}^{F \downarrow}=1.35 \AA^{-1}$. The values of the Fermi momenta define the positions of band bottoms $V_{1 \alpha}^{\mu}, V_{3 \alpha}^{\mu}(\alpha=s, d, \mu=\uparrow, \downarrow)$ with respect to $\varepsilon_{F}$. For $d$-electrons the Fermi momenta were chosen such a way that the interface density of states $\rho_{s(d)}^{\mu}\left(\varepsilon_{F}\right)=-1 / \pi \operatorname{Im} G_{s(d)}^{\mu}\left(z_{\alpha}\right)$ (where $z_{\alpha}=a, b$ are the positions of interfaces) comply with the following ratio $\rho_{s}^{\downarrow(\uparrow)}\left(\varepsilon_{F}\right): \rho_{d}^{\uparrow}\left(\varepsilon_{F}\right): \rho_{d}^{\downarrow}\left(\varepsilon_{F}\right) \sim 0.1: 1: 10$, which is the typical situation for the case of $3 d$ transition metals (see, for a example, the calculations of Tsymbal and Pettifor 14$)$. We also put the width of the barrier $w=20 \AA$, and $z_{0}=a_{0} / 2$ where $a_{0}=2.87 \AA$ is a lattice constant for bcc $\mathrm{Fe}\left(z_{0}\right.$ is a parameter describing the thickness of the interface).

The main features of the band structure of $\alpha-\mathrm{Al}_{2} \mathrm{O}_{3}$ crystals were briefly presented in Sec. IIA. In a view of that discussion, the following parameters of the model were taken to describe the amorphous $\mathrm{Al}_{2} \mathrm{O}_{3}$ barrier: the effective masses of electrons and holes are $m_{s}^{0}=0.4 m_{e}$, $m_{h}^{0}=-10.0 m_{e}$, the positions of the conduction band bottom $\left(U_{s}\right)$ and the top of a valence band $\left(U_{d}\right)$ are $U_{s}=$ $-U_{d}=3.0 \mathrm{eV}$, i.e. the width of the band gap is $\approx 6.0 \mathrm{eV}$, and $\varepsilon_{F}$ is assumed to be a zero of energy.

In order to illustrate the general formalism presented in the previous sections, let us first consider the case of only $s-d$ impurity scattering (i.e. $T=0$ and there are no spin-flip processes) when concentration of impurity ions on the interface is $(1-x)=0.5$. In this case all formulae have a simple analytical form. As it was mentioned previously in Sec. IIA, in the two-band tightbinding model description of a binary alloy the random variables are diagonal matrix elements of the Hamiltonian $\varepsilon_{n}^{s}(x), \varepsilon_{n}^{d}(x)$ and $\gamma_{n}(x)$ depending on whether the site $n$ is occupied by an A or B ion. To be more precise, one must consider the concentration dependence of these matrix elements. It reflects the fact that their values are modified by the existence of a charge transfer in the alloy. Since in the framework of our model we are focusing on the hybridization effects (see Sec. IIA for justification of this approach), we have $\gamma_{A}(x) \neq \gamma_{0}^{A}, \gamma_{B}(x) \neq \gamma_{0}^{B}$ where $\gamma_{0}^{A}$ and $\gamma_{0}^{B}$ are hybridizations of the pure components. Let us assume for simplicity that in Eq. (B2) $\gamma_{0}=\bar{\gamma}=x \gamma_{A}+y \gamma_{B}=0$. Then for $x=0.5$ we get $\gamma_{A}=-\gamma_{B}$, and thus $\gamma^{2}=x(1-x)\left(\gamma_{A}-\gamma_{B}\right)^{2}$ is the single parameter characterizing the amplitude of $s$ - $d$ scattering on the interface. The nonzero value of $\bar{\gamma}$ will lead to a slight modification of the $s$ and $d$-like eigenstates describing $s$ and $d$-like electrons in the vicinity of the interface, and thus will not affect the qualitative results presented below. We will omit spin suffixes since we consider the spin-conserving scattering now. At $x=0.5$ only diagonal elements of the self-energy matrix $\Sigma^{\alpha \beta}$ in the $s d$-space have non-zero values. Then the Green's functions, e.g. at the point $a$, become [see Eq. (21)]

$$
G^{s s}(a)=\int_{0}^{k_{\max }}\left\{\frac{k_{1}^{\mu s}}{2 m_{s}}\left(i-\operatorname{cotan} \varphi_{1}^{s}\right)\right.
$$




$$
\begin{aligned}
& \left.-\Sigma_{\mu}^{s s}(a)\right\}^{-1} \frac{\kappa d \kappa}{2 \pi} \\
G^{d d}(a)= & \int_{0}^{k_{\max }}\left\{\frac{k_{1}^{\mu d}}{2 m_{d}}\left[i-\operatorname{cotan}\left(k_{1}^{d} z_{0}+\varphi_{1}^{d}\right)\right]\right. \\
& \left.-\Sigma_{\mu}^{d d}(a)\right\}^{-1} \frac{\kappa d \kappa}{2 \pi}
\end{aligned}
$$

and CPA equations are written as follows

$$
\Sigma^{s s}=\frac{\gamma^{2} G^{d d}}{1+\Sigma^{d d} G^{d d}}, \quad \Sigma^{d d}=\frac{\gamma^{2} G^{s s}}{1+\Sigma^{s s} G^{s s}}
$$

which must be solved self-consistently by means of converging iterative procedure. As far as Green functions are diagonal, one can denote $\Gamma_{\alpha \beta}=\Gamma\left(\begin{array}{cc}\alpha & \beta \\ \alpha & \beta\end{array}\right)$ and can introduce the similar notations for quantities $T$ and $D$, defined in section IID. Then one gets

$$
\begin{gathered}
T_{s d}=T_{d s}=\frac{\gamma^{2}}{\left|1+\Sigma^{d d} G^{d d}\right|^{2}}=\frac{\gamma^{2}}{\left|1+\Sigma^{s s} G^{s s}\right|^{2}}, \\
T_{s s}=T_{d d}=0, \\
\Gamma_{s s}=\frac{T_{s d}^{2} D^{d d}}{1-T_{s d}^{2} D^{s s} D^{d d}}, \quad \Gamma_{d d}=\frac{T_{d s}^{2} D^{s s}}{1-T_{s d}^{2} D^{s s} D^{d d}}, \\
\Gamma_{s d}=\Gamma_{d s}=\frac{T_{s d}}{1-T_{s d}^{2} D^{s s} D^{d d}} .
\end{gathered}
$$

Since the mass of holes $m_{h}^{0}$ in the insulator is much larger than the electron mass $m_{s}^{0}$, the exponential factor $e^{-2 q_{2}^{d}(b-a)}$ in formulae 23 - 25) for tunneling conductance is negligibly small as compared with one for $s$-like electrons. Therefore, one may neglect the contribution from $d$-holes to the tunneling current. Then for the tunneling conductance we obtain

$$
\sigma=\sum_{\mu=\uparrow, \downarrow}\left(\sigma_{b}^{\mu}+\sigma_{\Gamma_{a}}^{\mu \mu}+\sigma_{\Gamma_{b}}^{\mu \mu}\right)
$$

where

$$
\sigma_{b}^{\mu}=\frac{e^{2}}{2 \pi \hbar} \int_{0}^{\kappa_{\max }} A_{\kappa \mu}^{s s}(a)\left(\frac{q_{2}^{s}}{m_{s}^{0}}\right)^{2} A_{\kappa \mu}^{s s}(b) e^{-2 q_{2}^{s} w} \frac{\kappa d \kappa}{2 \pi}
$$

is the conductance corresponding to the "bubble" diagram (here $w=b-a$ ),

$$
\begin{aligned}
\sigma_{\Gamma_{a}}^{\mu \mu} & =\frac{e^{2}}{2 \pi \hbar}\left(\int_{0}^{\kappa_{\max }} A_{\kappa \mu}^{d d}(a) \frac{\kappa d \kappa}{2 \pi}\right) \Gamma_{d s}^{(a) \mu \mu} \\
& \times\left[\int_{0}^{\kappa_{\max }}\left|G_{\kappa^{\prime} \mu}^{s s}(a)\right|^{2}\left(\frac{q_{2}^{s}}{m_{s}^{0}}\right)^{2} A_{\kappa^{\prime} \mu}^{s s}(b) e^{-2 q_{2}^{s} w} \frac{\kappa^{\prime} d \kappa^{\prime}}{2 \pi}\right] \\
& +\frac{e^{2}}{2 \pi \hbar}\left(\int_{0}^{\kappa_{\max }} A_{\kappa \mu}^{s s}(a) \frac{\kappa d \kappa}{2 \pi}\right) \Gamma_{s s}^{(a) \mu \mu} \\
& \times\left[\int_{0}^{\kappa_{\max }}\left|G_{\kappa^{\prime} \mu}^{s s}(a)\right|^{2}\left(\frac{q_{2}^{s}}{m_{s}^{0}}\right)^{2} A_{\kappa^{\prime} \mu}^{s s}(b) e^{-2 q_{2}^{s} w} \frac{\kappa^{\prime} d \kappa^{\prime}}{2 \pi}\right]
\end{aligned}
$$

is the "vertex" contribution to the conductance on the left interface, and

$$
\begin{aligned}
\sigma_{\Gamma_{b}}^{\mu \mu} & =\frac{e^{2}}{2 \pi \hbar}\left(\int_{0}^{\kappa_{\max }} A_{\kappa \mu}^{d d}(b) \frac{\kappa d \kappa}{2 \pi}\right) \Gamma_{d s}^{(b) \mu \mu} \\
& \times\left[\int_{0}^{\kappa_{\max }}\left|G_{\kappa^{\prime} \mu}^{s s}(b)\right|^{2}\left(\frac{q_{2}^{s}}{m_{s}^{0}}\right)^{2} A_{\kappa^{\prime} \mu}^{s s}(a) e^{-2 q_{2}^{s} w} \frac{\kappa^{\prime} d \kappa^{\prime}}{2 \pi}\right] \\
& +\frac{e^{2}}{2 \pi \hbar}\left(\int_{0}^{\kappa_{\max }} A_{\kappa \mu}^{s s}(b) \frac{\kappa d \kappa}{2 \pi}\right) \Gamma_{s s}^{(b) \mu \mu} \\
& \times\left[\int_{0}^{\kappa_{\max }}\left|G_{\kappa^{\prime} \mu}^{s s}(b)\right|^{2}\left(\frac{q_{2}^{s}}{m_{s}^{0}}\right)^{2} A_{\kappa^{\prime} \mu}^{s s}(a) e^{-2 q_{2}^{s} w} \frac{\kappa^{\prime} d \kappa^{\prime}}{2 \pi}\right]
\end{aligned}
$$

is the "vertex" contribution to the conductance on the right interface. Here $A_{\kappa \mu}^{\alpha \alpha}$ are the transport densities of states which for the present case on the left interface are given by the expressions

$$
\begin{aligned}
A_{\kappa \mu}^{s s}(a) & =\frac{k_{1}^{\mu s} / m_{s}}{\left|k_{1}^{s}\left(i-\operatorname{cotan} \varphi_{1}^{s}\right) / 2 m_{s}-\Sigma_{\mu}^{s s}(a)\right|^{2}} \\
A_{\kappa \mu}^{d d}(a) & =\frac{k_{1}^{\mu d} / m_{d}}{\left|k_{1}^{d}\left[i-\operatorname{cotan}\left(k_{1}^{d} z_{0}+\varphi_{1}^{d}\right)\right] / 2 m_{d}-\Sigma_{\mu}^{d d}(a)\right|^{2}} .
\end{aligned}
$$

and by the analogous expressions in the case of right interface.

We remind, that physically the "bubble" term in the total conductance is a contribution to the current due to direct tunneling when the electron momentum $\kappa=k_{\|}$in the plane of the interface is conserved. Scattering processes renormalize the "bubble" term with respect to the case of ballistic transport such that the effective "height" of the potential barrier seen by electron increases due to self-energy corrections $\Sigma_{\mu}^{s s}$ arising on the interfaces. The vertex contributions to the conductance describe the tunneling assisted by interfacial scattering — that is the processes of tunneling with scattering on the $\mathrm{F} / \mathrm{O}$ interface when the in-plane momentum is not conserved, i.e. $\kappa^{\prime} \neq \kappa$ for the scattered electron. Thus, both momenta $\kappa$ and $\kappa^{\prime}$ in Eq. (16) determine the vertex corrections.

Note also, that both vertex corrections $\sigma_{\Gamma_{a}}$ and $\sigma_{\Gamma_{b}}$ (42), (43) consist of two terms with vertices $\Gamma_{s d}$ and $\Gamma_{s s}$. The terms with vertex part $\Gamma_{s s}$ are contribution to the tunneling conductance from $s$-like electrons only. The scattering in the $s$ channel described by $\Sigma^{s s}(39)$ is caused initially by $s$ - $d$ scattering. The terms with $\Gamma_{s d}$ describe either the process of diffuse scattering of $d$-like electron to the $s$ state in the $\mathrm{F} / \mathrm{O}$ interface and then tunneling of $s$-like electron in the barrier, or the process of tunneling of $s$-electron and then its scattering to $d$ state in the $\mathrm{O} / \mathrm{F}$ interface and leaving into the electrode.

We have calculated the tunneling conductance and the TMR ratio defined as $\mathrm{TMR}=\left(\sigma_{P}-\sigma_{A P}\right) / \sigma_{A P}$, where $\sigma_{P}$ and $\sigma_{A P}$ are the total conductances for the parallel (P) and antiparallel (AP) alignment of magnetic moments in the F-layers. The CPA equations defining the self-energies were solved numerically. The validity of the 


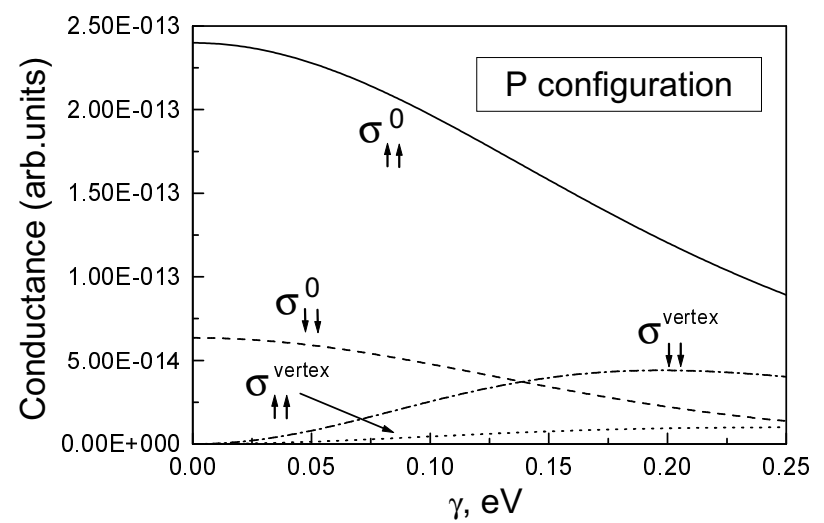

FIG. 4: "Bubble" and vertex contributions to the tunneling conductance (in the units $e^{2} / 2 \pi \hbar$ per unit square $1 \AA^{2}$ ) for the parallel $(\mathrm{P})$ alignment of magnetic moments in the ferromagnetic layers as a function of the scattering parameter $\gamma$ on the interface in the absence of spin-flip processes. The parameters of the model are: $s$-like electrons $-k_{\uparrow}^{F s}=1.09 \AA^{-1}$, $k_{\downarrow}^{F s}=0.42 \AA^{-1}, m_{s}=1.0, m_{s}^{0}=0.4 ; d$-like holes $k_{\uparrow}^{F d}=0.5 \AA^{-1}, k_{\downarrow}^{F d}=1.4 \AA^{-1}, m_{d}=m_{h}^{0}=-10.0$, the height of the potential barrier $U_{s}=-U_{d}=3.0 \mathrm{eV}$, the width of the barrier $w=20 \AA$, the concentration of Fe ions on the interface $x=0.5$.

Ward identity, Eq. (19), was checked after the vertex parts $\Gamma$ had been computed at every step for a given value of the parameter $\gamma$.

The results are presented in Figs. 4-7. In Fig. 4 the "bubble" (41) and the "vertex" (42), (43) contributions to the conductance for spin-up and spin-down channels are shown for the parallel $(\mathrm{P})$ alignment of magnetic moments as a function of the scattering parameter $\gamma$ on the interface. (We remind that up to now only the spinconserving scattering is considered.) As it was mentioned previously, scattering suppresses the "bubble" conductance, and hence $\sigma_{\uparrow \uparrow}^{0}$ and $\sigma_{\downarrow \downarrow}^{0}$ are decreasing functions on $\gamma$. The contribution from the "bubble" part is larger for the majority spin ( $\uparrow$ ) channel since $k_{\uparrow}^{F s}>k_{\downarrow}^{F s}$. On the contrary, the contribution from the vertex corrections dominates for the minority spin $(\downarrow)$ channel — that behavior can be understood as follows.

First, at small $\gamma^{2}$ the imaginary part of the self-energy $\Sigma^{s s}$, which describes the scattering of $s$-electrons, behaves as $\operatorname{Im} \Sigma_{\uparrow(\downarrow)}^{s s} \sim \pi \gamma^{2} \rho_{\uparrow(\downarrow)}^{d}\left(\varepsilon_{F}\right)$ [see Eq. (39)], where $\rho_{\uparrow(\downarrow)}^{d}\left(\varepsilon_{F}\right)$ are spin-up (down) $d$ density of states on the interface. As far as $\rho_{\downarrow}^{d}\left(\varepsilon_{F}\right)$ is of the order of magnitude greater than $\rho_{\uparrow}^{d}\left(\varepsilon_{F}\right)$, the scattering for $s$-like itinerant electrons from minority spin band $(\downarrow)$ is more effective than for the majority spin $(\uparrow)$ electrons. The more effective scattering of spin-down $s$-electons leads to the predominance of the minority spin contribution with vertex part $\Gamma_{s s}^{\downarrow}$ over the majority spin contribution with vertex

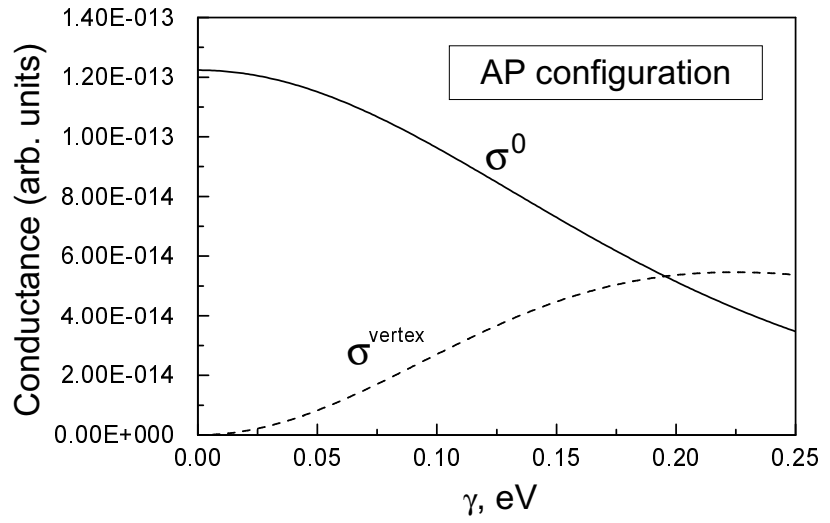

FIG. 5: "Bubble" and vertex contributions to the tunneling conductance (in the units $e^{2} / 2 \pi \hbar$ per unit square $1 \AA^{2}$ ) for the antiparallel (AP) alignment of magnetic moments as a function of the scattering parameter $\gamma$ on the interface in the absence of spin-flip processes. Parameters of the model are the same as in Fig. 4.

$\Gamma_{s s}^{\uparrow}$, since at small $\gamma, \Gamma_{s s}^{\uparrow(\downarrow)} \sim \gamma^{4} D_{\uparrow(\downarrow)}^{d d}$ and $D_{\downarrow}^{d d} \gg D_{\uparrow}^{d d}$, therefore $\Gamma_{s s}^{\downarrow} \gg \Gamma_{s s}^{\uparrow}$. Second, the transport density of states $A^{d d}(a)$ [Eq. (44)] which determines the partial conductance due to mixing of $s$ and $d$ channels in the interface (terms with $\Gamma_{s d}$ ) is also larger for minority spin channel. Thus, the combination of these factors results in domination of the vertex contribution to the conductance from the minority spin channel. The results of calculations also showed that the $s s$ contribution to the vertex corrections $\sigma_{\Gamma}^{s s}$ (terms with vertices $\Gamma_{s s}$ ) was much less than the $s d$ contribution $\sigma_{\Gamma}^{s d}$ (terms with $\Gamma_{s d}$ ).

The "bubble" and vertex contributions to the conductance (which are the same for spin-up and spin-down channels) for the antiparallel (AP) alignment of moments as a function of $\gamma$ are presented in Fig. 5. The total conductances for $\mathrm{P}$ and $\mathrm{AP}$ configurations are shown in Fig. 6. Finally, in Fig. 7 the TMR ratio $\left(\sigma_{P}-\sigma_{A P}\right) / \sigma_{A P}$ as a function of scattering parameter $\gamma$ is shown. When the amplitude of scattering is negligibly small $(\gamma=0)$, we have positive value of the TMR $\simeq 24 \%$ - that is the result of Slonczewski's theory 12 under the chosen parameters for $s$-like electrons. With increasing of $\gamma$ the TMR amplitude is monotonically decreasing and can become even negative if $\gamma>\gamma_{c} \approx 0.16 \mathrm{eV}$.

To understand qualitatively the obtained results, conductance and magnetoresistance can be rewritten in the general approximate form:

$$
\begin{aligned}
\sigma_{P} & \approx\left(\rho_{1}^{s \uparrow}\right)^{2}+\left(\rho_{1}^{s \downarrow}\right)^{2}+\rho_{3}^{d \uparrow} \widetilde{\Gamma}_{s d}^{\uparrow} \rho_{1}^{s \uparrow}+\rho_{3}^{d \downarrow} \widetilde{\Gamma}_{s d}^{\downarrow} \rho_{1}^{s \downarrow}, \\
\sigma_{A P} & \approx 2 \rho_{1}^{s \uparrow} \rho_{1}^{s \downarrow}+\rho_{3}^{d \uparrow} \widetilde{\Gamma}_{s d}^{\uparrow} \rho_{1}^{s \downarrow}+\rho_{3}^{d \downarrow} \widetilde{\Gamma}_{s d}^{\downarrow} \rho_{1}^{s \uparrow}, \\
\Delta \sigma & =\sigma_{P}-\sigma_{A P} \\
& \approx\left(\rho_{1}^{s \uparrow}-\rho_{1}^{s \downarrow}\right)^{2}+\left(\rho_{1}^{s \uparrow}-\rho_{1}^{s \downarrow}\right)\left(\rho_{3}^{d \uparrow} \widetilde{\Gamma}_{s d}^{\uparrow}-\rho_{3}^{d \downarrow} \widetilde{\Gamma}_{s d}^{\downarrow}\right),
\end{aligned}
$$




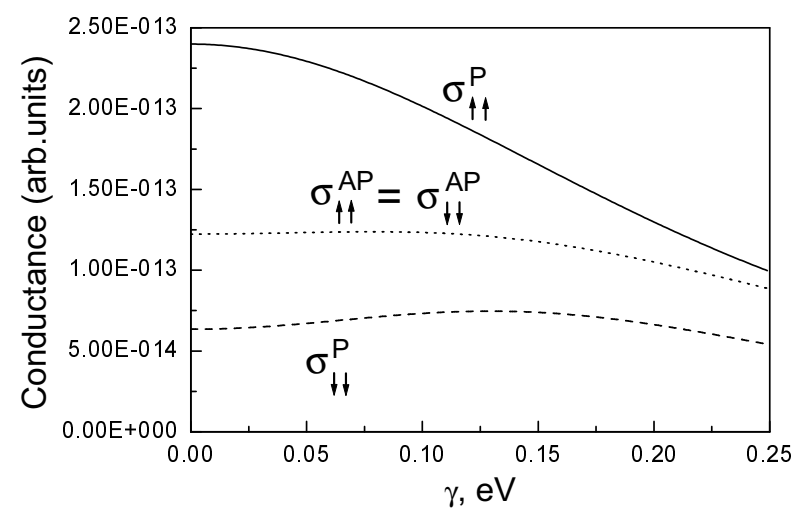

FIG. 6: The tunneling conductances of the individual spin channels (in the units $e^{2} / 2 \pi \hbar$ per unit square $1 \AA^{2}$ ) for the parallel (P) and antiparallel (AP) alignment of magnetic moments as the function of the scattering parameter $\gamma$ on the interface in the absence of spin-flip processes. The parameters are the same as in Fig. 4.

where we took into account that $\sigma_{\Gamma}^{s s} \ll \sigma_{\Gamma}^{s d}$, and for high and thick enough barrier the main contribution to the tunneling conductance is due to electrons with momentum almost perpendicular to the barrier (factors $e^{-2 q_{2}^{s} w}$ ). Consequently, $\rho_{1}^{s \uparrow(\downarrow)}$ are the quasi-one-dimensional $s$ density of states near the interface, and $\rho_{3}^{d \uparrow(\downarrow)}$ are the corresponding three-dimensional $d$ density of states. The $\widetilde{\Gamma}$ are renormalized vertex corrections. In expression (45) for $\Delta \sigma$, the first term due to direct tunneling is always positive. Concerning the second term, $\rho_{1}^{s \uparrow}>\rho_{1}^{s \downarrow}$ since $k_{F}^{s \uparrow}>k_{F}^{s \downarrow}$, but $\rho_{3}^{d \downarrow} \gg \rho_{3}^{d \uparrow}$ and, therefore, this term is negative. This contribution decreases the magnetoresistance when scattering parameter $\gamma$ becomes larger. At $\gamma>\gamma_{c}$ the $s d$ contribution overlaps the contribution to $\Delta \sigma$ from the ss channel, and we have TMR $<0$. Thus, the inverse TMR ratio arisen in our model is caused by extremely strong scattering of negatively polarized $d$-like electrons (which give the indirect contribution to the tunneling current) to the $s$-band on the interface.

The parameter $\gamma$ determining the amplitude of $s$ - $d$ scattering on the interface is defined as $\gamma^{2}=x(1-x)\left(\gamma_{A^{-}}\right.$ $\left.\gamma_{B}\right)^{2}$. One can regard the difference between hybridizations, $\gamma_{A}-\gamma_{B}$, as approximately a constant value for given constituents. However, $(1-x)$ is a concentration of impurity centers, and thus $\gamma$ is a measure of the imperfection of the F/O interface. The proposed model, therefore, explains qualitatively the strong interface sensitivity of the tunneling magnetoresistance effect 0 . According to Heine's discussion of the hybridization in transition metals37, the hybridization constants are from about 1.0 to $3.0 \mathrm{eV}$ for different elements. One can assume that the difference $\left(\gamma_{A}-\gamma_{B}\right)$ is of the order of magnitude smaller and, therefore, the critical value of scattering parameter $\gamma_{c} \simeq 0.16 \mathrm{eV}$ that we obtained is more or less realistic.

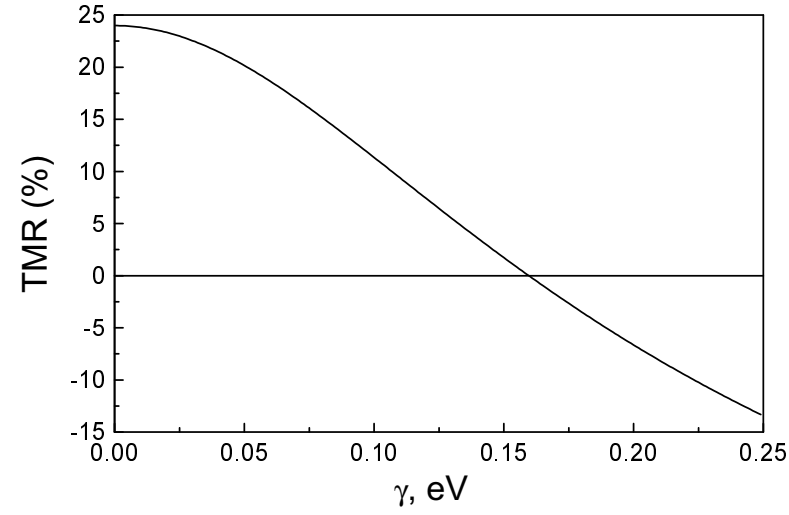

FIG. 7: The tunneling magnetoresistance (TMR) as a function of the scattering parameter $\gamma$ in the case of only elastic scattering on the interfaces. The parameters are the same as in Fig. 4.

The strong reduction of the TMR due to nonideal structure of the metal/insulator interfaces is a wellknown observation. The oxidation of a thin Al layer leads to the undesirable oxidation of few metal monolayers close to the $\mathrm{F} / \mathrm{O}$ interface and thus to the formation of $\mathrm{F}-\mathrm{O}$ oxides $\left(\mathrm{Fe}_{\beta} \mathrm{O}_{4} \mathrm{LO}, \mathrm{CoO}\right.$ and $\mathrm{Co}_{3} \mathrm{O}_{4}$ 是) that reduces the TMR 10 . On the other hand, if the too thick Al layer is not oxidized completely, the contamination of the interface by $\mathrm{Al}$ ions also reduces the TMR 10 . The dependence of the TMR vs. the thickness of the $\mathrm{Al}$ overlayer has, therefore, a maximum and the best TMR valuesachieved by Moodera's group lie in the range $10 \div 16 \AA$.

The contamination of the $\mathrm{F} / \mathrm{O}$ interface by $\mathrm{OH}$ ions in the early experiments by Merserwey and Tedrow on tunneling with the superconductors 5 led to the small measured values of a spin polarization $P$ for $\mathrm{Ni}$ and $\mathrm{Gd}$. The contamination was due to oxidizing of the $\mathrm{Al}$ films in the laboratory air. The improved technique of samples preparation in a pure oxygen increased the valpes of $P$ for $\mathrm{Ni}$ and $\mathrm{Gd}$, and for some rare-earth metals 5 -

In a recent work by LeClair et al 30 the strong suppression of magnetoresistance was observed in $\mathrm{Co} / \mathrm{Al}_{2} \mathrm{O}_{3} / \mathrm{Co}$ tunnel junctions with a very thin $\mathrm{Cr}$ interfacial layer. It was found that the TMR decayed exponentially on the Cr interlayer thickness with a length scale $\sim 1 \AA$ (approximately 0.5 monolayers). With the addition of only $3 \AA$ $\mathrm{Cr}(\approx 1.5$ monolayers $)$ the reduced TMR was only $10 \%$ of the initial value for a control junction. LeClair et al. 3 presented some qualitative arguments that the suppression of a spin polarization (and, hence, the reduction of TMR) was due to more strongly suppression of majority $s-p$ density of states compared with minority spins caused by the resonant scattering of majority spin $s p$ electrons with the $\mathrm{Cr} d$ states. From the point of view of our model, we can explain the strong degradation of the 


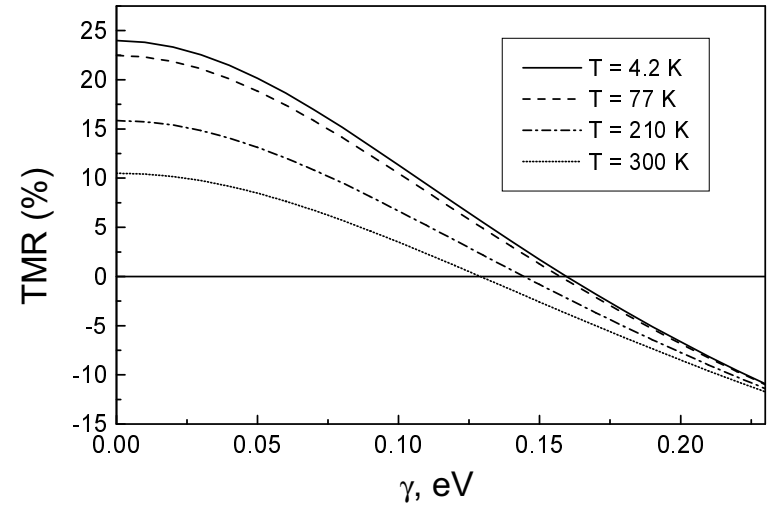

FIG. 8: The TMR for different temperatures as a function of the scattering parameter $\gamma$.

TMR by the strong electron scattering within the interfacial Co-Cr alloy that is formed under the preparation of extremely thin ( $\sim 1$ monolayer $) \mathrm{Cr}$ interlayer.

We have also calculated the temperature dependence of the TMR taking into account spin-flip scattering in addition to $s-d$ impurity scattering as it is described in details in sections IIC and IID. For that, the average magnon number $n(T)$ (33) as a function of the temperature was found in analogy with Debye's treatment of phonons using the similar approach that was proposed by $\mathrm{S}$. Zhang et a 13 . The magnon dispersion relation in Eq. (33) was replaced by simple isotropic parabolic spectrum

$$
\omega_{\mathbf{q}}=E_{m}\left(\frac{q}{k_{\max }}\right)^{2}
$$

where $k_{\max }$ is the equivalent radius of the two-dimensional Brillouin zone (see Eq. 30), and $E_{m}$ is related to

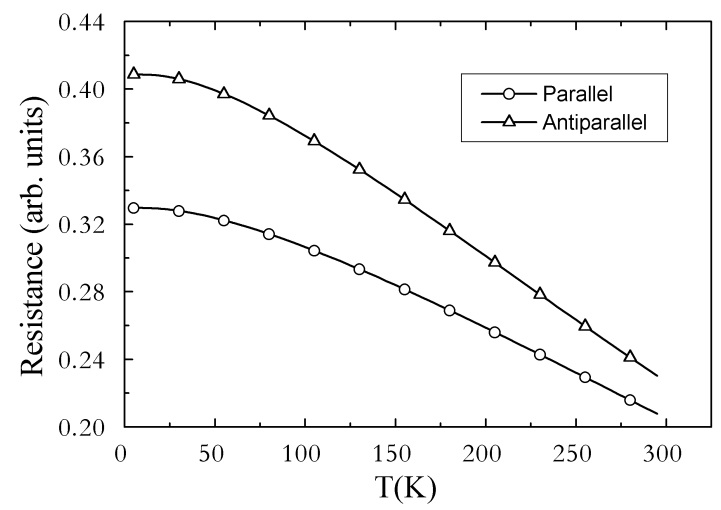

FIG. 9: The temperature dependence of the resistance for $\mathrm{P}$ and AP configurations of the magnetic moments at $\gamma=0$ and $x=50 \%$.
Curie temperature $T_{c}$ and in the mean-field approximation is given by $E_{m}=3 k_{B} T_{c} /(S+1)$. For the chosen model of the dispersion relation $w_{\mathbf{q}}$, one has to overcome the divergence on the lower limit of the integral in Eq. (33). Therefore, one must introduce a lower wavelength cutoff $E_{c}$ 13. Physically, it may represent a finite coherence length due to interfacial roughness. In our calculation we have taken the same parameters that were used by S. Zhang et al. 13 for the analysis of the zero-bias anomaly: $S=3 / 2, k_{B} T_{c}=110 \mathrm{meV}$, and $E_{c}=4 \mathrm{meV}$. Then, for the temperature range within the room value, we have

$$
n(T)=-\frac{1}{2}\left(\frac{k_{B} T}{E_{m}}\right) \log \left(1-e^{-E_{c} / k_{B} T}\right) .
$$

We have put $x=50 \%, J_{A}=2.0 \mathrm{eV}$ (for Fe atoms) and $J_{B}=0 \mathrm{eV}$, i.e. it is supposed that spin-flip process is possible, if an electron scatters on the $\mathrm{Fe}$ ion. The TMR vs. the scattering parameter $\gamma$ at different temperatures $T=4.2,77,210$ and $300 \mathrm{~K}$ are plotted in Fig. 8 . In Fig. 9 the temperature dependence of the resistance for the $\mathrm{P}$ and $\mathrm{AP}$ configuration of magnetizations is presented for the same parameters, but $\gamma=0$. The results show that the TMR ratio decreases with increasing of the temperature. Moreover, the resistances $R_{P}$ and $R_{A P}$ of the structure for both configurations, $\mathrm{P}$ and $\mathrm{AP}$, are also decreasing when temperature increases - that is in the qualitative agreement with experimental data (e.g., see Fig. 4b in Ref. [38]). The physical mechanism of this effect is related with the excitation of spin-flip processes in the system. Due to these processes, the new channels of electron scattering appear which are frozen at zero temperature. As the result, the conductance of the system increases for both $\mathrm{P}$ and AP configurations and, therefore, the resistance drops. The spin-flip processes mix the spin-up and spin-down channels. Therefore, the relative difference of the resistances decreases at different configurations and the TMR decreases with increasing of temperature.

\section{CONCLUSIONS}

In conclusion, we would like to outline the main results obtained in the present work. Based on the analysis of the band structure of $3 d$ ferromagnetic metals and $\mathrm{Al}_{2} \mathrm{O}_{3}$ crystals and on the results of ab-initio calculations of the magnetoresistance for epitaxial tunnel junctions, we built on an adaptation of the simplified two-band $s$ - $d$ model to treat the diffuse electron transport in the nonideal $\mathrm{F} / \mathrm{O} / \mathrm{F}$ magnetic tunnel junctions. We had modeled the rough $\mathrm{F} / \mathrm{O}$ interface by the random binary alloy that is formed from the ions of the ferromagnet and impurities (e.g. the $\mathrm{Al}$ or $\mathrm{O}$ ions), and assumed that the main mechanism of electron scattering on the interface which substantially affects tunneling is the $s$ - $d$ scattering. We used the Kubo formalism to calculate the tunneling conductance and found the vertex corrections to 
conductivity with the use of the "ladder" approximation combined with the CPA. The obtained results show that in the case of strong electron scattering within the interfacial alloy the vertex corrections give the essential contribution to the tunneling conductance. We proved that adopted approximations lead to the physically correct results, namely, the non-local conductivity tensor is a constant function and, therefore, a tunneling current is conserved. We showed that interfacial inter-band scattering substantially reduces a value of the TMR, which can become even negative in the case of extremely strong scattering. The reason of the suppression of the magnetoresistance is the indirect contribution of negatively polarized $d$-like electrons to the tunneling current due to strong scattering to the $s$ band on the interface. It is also shown that spin-flip electron scattering on the surface magnons within the interface leads to a further decrease of the TMR at finite temperature. Thus, in the framework of the proposed model, we are able to explain qualitatively the strong interface sensitivity of the tunneling properties that is observed in the experiments.

\section{Acknowledgments}

A. Vedyayev and D. Bagrets are grateful to CEA/Grenoble/DRFMC/SP2M/NM for fellowships, A. Bagrets is grateful to Laboratoire Louis Néel/CNRS (Grenoble, France) for hospitality. This work was partially supported by Russian Foundation for Basic Research (grant No. 98-02-16806).

\section{APPENDIX A: WARD IDENTITY}

In this Appendix we briefly describe how to obtain Eq. (19) (Ward identity), and using a simple example we will show how this identity can be proved.

Starting from Eq. (20), one first has to compute the derivation of the "bubble" conductivity. Assuming, that the self-energy $\Sigma_{\mu}^{\alpha \beta}$ is symmetric with respect to the rearrangement of band indices, i.e. $\Sigma_{\mu}^{\alpha \beta}=\Sigma_{\mu}^{\beta \alpha}$, and using the fact that functions $\psi_{i}^{\alpha}\left(z^{\prime}\right)$ are the solutions of the Schrödinger equation (7), for the derivation of the current matrix we obtain:

$$
\frac{\partial}{\partial z^{\prime}} j_{\mu}^{\psi}\left(z^{\prime}\right)=\sum_{\alpha \beta=s, d} 2 \operatorname{Im} \Sigma_{\mu}^{\alpha \beta}\left(z^{\prime}\right)\left[\rho_{\mu}^{\psi}\left(z^{\prime}\right)\right]^{\alpha \beta}
$$

where

$$
\Sigma_{\mu}^{\alpha \beta}\left(z^{\prime}\right)=\Sigma_{\mu}^{\alpha \beta}(a) \delta\left(z^{\prime}-a\right)+\Sigma_{\mu}^{\alpha \beta}(b) \delta\left(z^{\prime}-b\right),
$$

and the matrix $\left[\rho_{u}^{\psi}\left(z^{\prime}\right)\right]^{\alpha \beta}$ is defined by Eq. (18). Then, from Eq. (15), (17) we have

$$
\frac{\partial}{\partial z^{\prime}} \sigma_{\mu}^{0}\left(z, z^{\prime}\right)=-\frac{e^{2}}{2 \pi \hbar A} \sum_{\kappa} \sum_{\alpha \beta=s, d}\left[2 \operatorname{Im} \Sigma_{\mu}^{\alpha \beta}(a)\right.
$$

$$
\begin{aligned}
& \times \delta\left(z^{\prime}-a\right) \Lambda_{\mu \kappa}^{\alpha \beta}(z, a) \\
& \left.+2 \operatorname{Im} \Sigma_{\mu}^{\alpha \beta}(b) \delta\left(z^{\prime}-b\right) \Lambda_{\mu \kappa}^{\alpha \beta}(z, b)\right]
\end{aligned}
$$

To proceed further, let us compute the derivation of the vertex correction to conductivity. The initial expression for the matrix $\Lambda_{\mu \kappa}$ (see diagram in Fig. 2) is written as

$$
\Lambda_{\mu \kappa}^{\gamma_{1} \gamma_{2}}\left(a, z^{\prime}\right)=\sum_{\alpha=s, d} G_{\mu \kappa}^{* \gamma_{1} \alpha}\left(a, z^{\prime}\right) \frac{\stackrel{\leftrightarrow}{\nabla}_{z^{\prime}}}{2 i m_{\alpha}} G_{\mu \kappa}^{\alpha \gamma_{2}}\left(z^{\prime}, a\right)
$$

Taking into account that the Green's functions are the solution of the Eq. (5), we obtain

$$
\begin{aligned}
\frac{\partial}{\partial z^{\prime}} \Lambda_{\mu \kappa}^{\gamma_{1} \gamma_{2}}\left(a, z^{\prime}\right)= & -2 \delta\left(z^{\prime}-a\right) \operatorname{Im} G_{\mu \kappa}^{\gamma_{1} \gamma_{2}}(a) \\
& +2 \sum_{\alpha \beta=s, d} \operatorname{Im} \Sigma_{\mu}^{\alpha \beta}(a) G_{\mu \kappa}^{* \alpha \gamma_{1}}(a) \\
& \times G_{\mu \kappa}^{\beta \gamma_{2}}(a) \delta\left(z^{\prime}-a\right) .
\end{aligned}
$$

Substituting the obtained expression to formula (16) for the vertex correction, we get

$$
\begin{aligned}
\frac{\partial}{\partial z^{\prime}} \sigma_{\mu \rho}^{\Gamma_{a}}\left(z, z^{\prime}\right)= & \frac{e^{2}}{2 \pi \hbar A^{2}} \sum_{\kappa \kappa^{\prime}} 2 \delta\left(z^{\prime}-a\right) \Lambda_{\mu \kappa}^{\beta_{1} \beta_{2}}(z, a) \\
& \times \Gamma^{\mu \rho}\left(\begin{array}{cc}
\beta_{1} & \gamma_{1} \\
\beta_{2} & \gamma_{2}
\end{array}\right)\left[\operatorname{Im} G_{\rho \kappa^{\prime}}^{\gamma_{1} \gamma_{2}}(a) \quad(\mathrm{A} 2)\right. \\
& \left.-\operatorname{Im} \Sigma_{\rho}^{\alpha_{1} \alpha_{2}}(a) G_{\rho \kappa^{\prime}}^{* \alpha_{1} \gamma_{1}}(a) G_{\rho \kappa^{\prime}}^{\alpha_{2} \gamma_{2}}(a)\right] .
\end{aligned}
$$

The summation here is also performed over indices $\alpha_{i}$, $\beta_{i}$, and $\gamma_{i}$. A similar expression can be written for the derivation of the vertex correction $\sigma_{\mu \rho}^{\Gamma_{b}}\left(z, z^{\prime}\right)$ at interface $z=b$. From (A1) and (A2) one can obtain the final expression (19) for Ward identity.

Let's prove identity for the simple case of only $s-$ $d$ scattering, when there are no spin-flip processes, and $x=0.5$. This situation was considered in Sec. III, and there were introduced the notations $\Gamma_{\alpha \beta}, T_{\alpha \beta}$, and $D^{\alpha \beta}$ $(\alpha \beta=s, d)$ for the components of the $(2 \times 2)$-matricies $\Gamma, T$, and $D$. Within these notations, the identity (19), which has to be proved, can be written as

$$
\operatorname{Im} \Sigma^{\alpha \alpha}=\sum_{\beta=s, d} \Gamma_{\alpha \beta}\left[\operatorname{Im} G^{\beta \beta}-\frac{1}{S} \sum_{\kappa}\left|G_{\kappa}^{\beta \beta}\right|^{2} \operatorname{Im} \Sigma^{\beta \beta}\right] .
$$

Here we omitted spin suffixes, all values associated either with the interface $a$ or $b, G^{\beta \beta}$ and $\Gamma_{\alpha \beta}$ are defined by Exps. (38, 39). From (36, 37) it follows that

$$
D^{\alpha \alpha}=\frac{1}{A} \sum_{\kappa}\left|G_{\kappa}^{\alpha \alpha}\right|^{2}-\left|G^{\alpha \alpha}\right|^{2}, \quad \Gamma^{-1}=T^{-1}-D .
$$

Using these expressions, Ward identity can be written in the form:

$$
\operatorname{Im} G^{\alpha \alpha}-\sum_{\beta=s, d}\left\{\left[T^{-1}\right]_{\alpha \beta}+\delta_{\alpha \beta}\left|G^{\alpha \alpha}\right|^{2}\right\} \operatorname{Im} \Sigma^{\beta \beta}=0 .
$$


Using (40), we then can find that

$$
\begin{aligned}
\operatorname{Im} \Sigma^{s s}= & \frac{1}{\operatorname{Den}}\left(\left|G^{d d}\right|^{2} \operatorname{Im} G^{s s}\right. \\
& \left.+\gamma^{-2}\left|1+\Sigma^{s s} G^{s s}\right|^{2} \operatorname{Im} G^{d d}\right), \\
\operatorname{Im} \Sigma^{d d}= & \frac{1}{\operatorname{Den}}\left(\left|G^{s s}\right|^{2} \operatorname{Im} G^{d d}\right. \\
& \left.+\gamma^{-2}\left|1+\Sigma^{d d} G^{d d}\right|^{2} \operatorname{Im} G^{s s}\right),
\end{aligned}
$$

where

$$
\text { Den }=\left|G^{s s}\right|^{2}\left|G^{d d}\right|^{2}-\gamma^{-4}\left|1+\Sigma^{s s} G^{s s}\right|^{4} .
$$

The same expessions for $\operatorname{Im} \Sigma^{s s}$ and $\operatorname{Im} \Sigma^{d d}$ follow directly from the CPA equations (39) - that finishes the proof.

\section{APPENDIX B: THE DERIVATION OF THE CPA EQUATIONS}

In this Appendix we will derive the CPA equations for our particular case using the augmented-space formalism (ASF) B. As was described previously, we assume the $\mathrm{F} / \mathrm{O}$ interface to be a random binary alloy of the type $\mathrm{A}_{x} \mathrm{~B}_{1-x}$, where $\mathrm{A}$ are ions of the ferromagnet and $\mathrm{B}$ are impurities. Following the ASF, we associate each random variable $\gamma_{n}^{\alpha}$ and $J_{n}^{\alpha}$ with the self-conjugate operators $\tilde{\gamma}$ and $\tilde{J}$, respectively, which are determined in the auxiliary 2-dimensional vector space $\Phi$ such a way, that the spectrum of these operators coincides with the set of possible values of random variables. For the sake of clarity, hereafter, the sign "tilda" is ascribed to any operator acting on the auxiliary space. We also define the orthonormalized basis $|s\rangle$, where $s=A$ or $B$, which are eigenvectors of $\tilde{\gamma}$ and $\tilde{J}$, so that

$$
\begin{aligned}
& \tilde{\gamma}|A\rangle=\gamma_{A}|A\rangle, \quad \tilde{J}|A\rangle=J_{A}|A\rangle, \\
& \tilde{\gamma}|B\rangle=\gamma_{B}|B\rangle, \quad \tilde{J}|B\rangle=J_{B}|B\rangle .
\end{aligned}
$$

According to that definition, $\tilde{\gamma}$ and $\tilde{J}$ commutate with each other. Let now $f\left(\gamma_{n}^{\alpha}, J_{n}^{\alpha}\right)$ be a function or an operator of random variables $\gamma_{n}^{\alpha}$ and $J_{n}^{\alpha}$. Then, the operator in the auxiliary space $\Phi$, associated with function $f$, is defined as $\tilde{f}=f(\tilde{\gamma}, \tilde{J})$ and according to (B1), e.g. $\langle A|\tilde{f}| A\rangle$ is a value of $f$, if the site $n$ is occupied by an ion A. One can introduce another orthonormal basis in $\Phi$

$$
|0\rangle=\sqrt{x}|A\rangle+\sqrt{y}|B\rangle, \quad|1\rangle=\sqrt{y}|A\rangle-\sqrt{x}|B\rangle,
$$

so that the operators $\tilde{\gamma}$ and $\tilde{J}$ in this representation are written as

$$
\tilde{\gamma}=\left(\begin{array}{cc}
\gamma_{0} & \gamma \\
\gamma & \gamma_{1}
\end{array}\right), \quad \tilde{J}=\left(\begin{array}{cc}
J_{0} & \delta \\
\delta & J_{1}
\end{array}\right) .
$$

Here $x$ and $y$ are the concentration of A-type ions (ferromagnet's ions) and B-type ions (impurities) on the interface, respectively, and

$$
\begin{aligned}
\gamma_{0}=x \gamma_{A}+y \gamma_{B}, & \gamma_{1}=y \gamma_{A}+x \gamma_{B}, \\
\gamma=\sqrt{x y}\left(\gamma_{A}-\gamma_{B}\right), & J_{0}=x J_{A}+y J_{B}, \\
J_{1}=y J_{A}+x J_{B}, & \delta=\sqrt{x y}\left(J_{A}-J_{B}\right) .
\end{aligned}
$$

Then one can prove that the average value of $f$ is given by $\bar{f}=\langle 0|\tilde{f}| 0\rangle$. Together with Eq. (B2) this property is the way to evaluate the average of any given operator, depending on the random variables, and we can apply this method to average the $t$-matrix (28).

Following the general scheme of the ASF, outlined above, the random effective potential $\hat{u}_{n}^{\alpha}=\hat{v}_{n}^{\alpha}-\hat{\Sigma}^{\alpha}$ is associated with the operator $\tilde{U}-\tilde{\Sigma}$ acting in the augmented vector space $\Phi \otimes L$, where $L$ denotes the 4 -dimensional space of orbital $(s, d)$ and spin $(\uparrow, \downarrow)$ electron degrees of freedom. In accordance with (26), (27) and (B2) this operator has the form

$$
\begin{aligned}
\tilde{U}-\tilde{\Sigma} & =\left(\begin{array}{cc}
\hat{U}_{0}-\hat{\Sigma} & \hat{\Delta} \\
\hat{\Delta} & \hat{U}_{1}-\hat{\Sigma}
\end{array}\right) \\
& =\left(\begin{array}{cc}
\hat{\gamma}_{0}+\hat{J}_{0} \hat{v}-\hat{\Sigma} & \hat{\gamma}+\hat{\delta} \hat{v} \\
\hat{\gamma}+\hat{\delta} \hat{v} & \hat{\gamma}_{1}+\hat{J}_{1} \hat{v}-\hat{\Sigma}
\end{array}\right)
\end{aligned}
$$

where $\hat{\Sigma}$ is defined by (29), operators $\hat{\gamma}_{(i)}$ are defined similar to (26), and other operators are given by

$$
\begin{aligned}
& \hat{v}=|\uparrow\rangle \hat{S}_{-}\left(\rho_{n}^{\alpha}\right)\langle\downarrow|+| \downarrow\rangle \hat{S}_{+}\left(\rho_{n}^{\alpha}\right)\langle\uparrow|, \\
& \hat{\delta}=\delta|s\rangle\left\langle s\left|, \quad \hat{J}_{i}=J_{i}\right| s\right\rangle\langle s| \quad(i=0,1) .
\end{aligned}
$$

Let us also introduce the nonrandom averaged propagator acting in the augmented space

$$
\tilde{G}=\left(\begin{array}{cc}
\hat{G} & 0 \\
0 & \hat{G}
\end{array}\right)
$$

and associated with potential $\tilde{U}$ (B3) the augmented scattering $t$-matrix

$$
\tilde{t}=(\tilde{U}-\tilde{\Sigma}) \frac{1}{1-\tilde{G}(\tilde{U}-\tilde{\Sigma})}=\left(\begin{array}{cc}
\hat{t}_{00} & \hat{t}_{01} \\
\hat{t}_{10} & \hat{t}_{11}
\end{array}\right) .
$$

Its projection onto the zero-level $|0\rangle$ of the augmented space $\hat{t}_{00}=\langle 0|\tilde{t}| 0\rangle$ coincides with the average from the "physical" random $t$-matrix (28). The subsequent averaging over magnon degrees of freedom $\left\langle t_{00}\right\rangle_{b}$ must vanish due to condition (34).

To proceed further, let's introduce the electron propagator $\hat{G}_{1}$

$$
\hat{G}_{1}=\frac{1}{\hat{G}^{-1}-\left(\hat{U}_{1}-\hat{\Sigma}\right)}=\left(\begin{array}{cc}
\hat{G}_{1}^{\uparrow} & \hat{G}_{1}^{+} \\
\hat{G}_{1}^{-} & \hat{G}_{1}^{\downarrow}
\end{array}\right),
$$

which is associated with the "propagation" of an electron on the first level of the augmented space in the potential 
$\hat{U}_{1}-\hat{\Sigma}$. Taking into account the explicit form of $\hat{U}_{1}-\hat{\Sigma}$ with respect to spin-up and spin-down subspaces,

$$
\hat{U}_{1}-\hat{\Sigma}=\left(\begin{array}{cc}
\hat{\gamma}_{1}-\hat{\Sigma}^{\uparrow} & \hat{J}_{1} \hat{S}_{-} \\
\hat{J}_{1} \hat{S}_{+} & \hat{\gamma}_{1}-\hat{\Sigma}^{\downarrow}
\end{array}\right)
$$

where operators $\hat{\gamma}_{1}$ and $\hat{J}_{1}$ are

$$
\begin{aligned}
& \hat{\gamma}_{1}=\gamma_{1}\{|s\rangle\langle d|+| d\rangle\langle s|\}, \\
& \hat{J}_{1}=J_{1}|s\rangle\langle s|,
\end{aligned}
$$

one gets

$$
\begin{aligned}
& \hat{G}_{1}^{\uparrow}=\left[1-\hat{G}^{\uparrow}\left(\hat{\gamma}_{1}-\Sigma^{\uparrow}+\hat{J}_{1} \hat{n}_{+} \hat{g}_{1}^{\downarrow} \hat{J}_{1}\right)\right]^{-1} \hat{G}^{\uparrow}, \\
& \hat{G}_{1}^{\downarrow}=\left[1-\hat{G}^{\downarrow}\left(\hat{\gamma}_{1}-\Sigma^{\downarrow}+\hat{J}_{1} \hat{n}_{-} \hat{g}_{1}^{\uparrow} \hat{J}_{1}\right)\right]^{-1} \hat{G}^{\downarrow}, \\
& \hat{G}_{1}^{+}=\hat{G}_{1}^{\uparrow} \hat{J}_{1} \hat{S}_{-} \hat{g}_{1}^{\downarrow}, \quad \hat{G}_{1}^{-}=\hat{G}_{1}^{\downarrow} \hat{J}_{1} \hat{S}_{+} \hat{g}_{1}^{\uparrow},
\end{aligned}
$$

where

$$
\hat{g}_{1}^{\uparrow(\downarrow)}=\left[1-\hat{G}^{\uparrow(\downarrow)}\left(\hat{\gamma}_{1}-\hat{\Sigma}^{\uparrow(\downarrow)}\right)\right]^{-1} \hat{G}^{\uparrow(\downarrow)},
$$

and $\hat{n}_{+}=\hat{S}_{-} \hat{S}_{+}, \hat{n}_{-}=\hat{S}_{+} \hat{S}_{-}$. The physical meaning of these formulae is rather transparent. The Green's function $g_{1}^{\uparrow \downarrow)}$ corresponds to the propagation of the electron in a spin conserving part of the potential $\hat{U}_{1}-\hat{\Sigma}$ which is $\hat{\gamma}_{1}-\hat{\Sigma}^{\uparrow(\downarrow)}$, while $\hat{G}_{1}^{\uparrow()}$ corresponds to scattering on the potential $\hat{\gamma}_{1}-\hat{\Sigma}^{\uparrow(\downarrow)}+\hat{J}_{1} \hat{n}_{ \pm} \hat{g}_{1}^{\downarrow(\uparrow)} \hat{J}_{1}$, renormalized with respect to the spin-conserving potential due to the interaction with surface magnons on the interface.

Coming back to the evaluation of scattering matrix element $\hat{t}_{00}$, let introduce the "denominator" $\tilde{D}$, corresponding to the whole augmented potential $\tilde{U}-\tilde{\Sigma}$

$$
\tilde{D}=\frac{1}{1-\tilde{G}(\tilde{U}-\tilde{\Sigma})}=\left(\begin{array}{cc}
\hat{D}_{00} & \hat{D}_{01} \\
\hat{D}_{10} & \hat{D}_{11}
\end{array}\right)
$$

Again, using the technique of the inversion of a matrix in the block form and taking into account the elements $\tilde{U}$ (B3) with respect to the auxiliary space $\Phi$, the blocks of $\tilde{D}$ can be expressed in terms of the propagator $\hat{G}_{1}$ as follows

$$
\begin{aligned}
& \hat{D}_{00}=\left[1-\hat{G}\left(\hat{U}_{0}-\hat{\Sigma}+\hat{\Delta} \hat{G}_{1} \hat{\Delta}\right)\right]^{-1}, \\
& \hat{D}_{10}=\hat{G}_{1} \hat{\Delta} \hat{D}_{00}, \quad \hat{D}_{01}=\hat{G}_{0} \hat{\Delta} \hat{D}_{1}, \\
& \hat{D}_{11}=\left(1+\hat{G}_{0} \hat{\Delta} \hat{G}_{1} \hat{\Delta}\right) \hat{D}_{00},
\end{aligned}
$$

where we define the propagator $\hat{G}_{0}$,

$$
\hat{G}_{0}=\left[1-\hat{G}\left(\hat{U}_{0}-\hat{\Sigma}+\hat{\Delta} \hat{G}_{1} \hat{\Delta}\right)\right]^{-1} \hat{G}=\left(\begin{array}{cc}
\hat{G}_{0}^{\uparrow} & \hat{G}_{0}^{+} \\
\hat{G}_{0}^{-} & \hat{G}_{0}^{\downarrow}
\end{array}\right),
$$

corresponding to the propagation of the electron in the effective potential $\hat{W}-\hat{\Sigma}$, where

$$
\hat{W}=\hat{U}_{0}+\hat{\Delta} \hat{G}_{1} \hat{\Delta}=\left(\begin{array}{cc}
\hat{w}^{\uparrow} & \hat{J}_{+} \hat{S}_{-} \\
\hat{J}_{-} \hat{S}_{+} & \hat{w}^{\downarrow}
\end{array}\right) .
$$

The potential $\hat{W}$ can be regarded as a renormalization of the "virtual" crystal potential $\hat{U}_{0}$ of the zero level of the augmented space, representing the average of the random potential on a site. The renormalization comes from the "interaction" $\hat{\Delta}$ in the auxiliary space with the first level, being described by the propagator $\hat{G}_{1}$. Using the explicit form of $\hat{\Delta}$.

$$
\hat{\Delta}=\left(\begin{array}{cc}
\hat{\gamma} & \hat{\delta} \hat{S}_{-} \\
\hat{\delta} \hat{S}_{+} & \hat{\gamma}
\end{array}\right)
$$

one can write down the elements $\hat{w}^{\uparrow(\downarrow)}$ and $\hat{J}_{ \pm}$:

$$
\begin{aligned}
\hat{w}^{\uparrow(\downarrow)}= & \hat{\gamma}_{0}+\hat{\gamma} \hat{G}_{1}^{\uparrow()} \hat{\gamma}+\hat{\gamma} \hat{G}_{1}^{\uparrow(\downarrow)} \hat{J}_{1} \hat{n}_{ \pm} \hat{g}_{1}^{\downarrow(\uparrow)} \hat{\delta} \\
& +\hat{\delta} \hat{G}_{1}^{\downarrow(\uparrow)} \hat{J}_{1} \hat{n}_{ \pm} \hat{g}_{1}^{\uparrow()} \hat{\gamma}+\hat{\delta} \hat{n}_{ \pm} \hat{G}_{1}^{\downarrow(\uparrow)} \hat{\delta}, \\
\hat{J}_{ \pm}= & \hat{J}_{0}+\hat{\gamma} \hat{G}_{1}^{\uparrow(\downarrow)} \hat{\delta}+\hat{\gamma} \hat{G}_{1}^{\uparrow(\downarrow)} \hat{J}_{1} \hat{g}_{1}^{\downarrow(\uparrow)} \hat{\gamma} \\
& +\hat{\delta} \hat{G}_{1}^{\downarrow(\uparrow)} \hat{J}_{1} \hat{n}_{ \pm} \hat{g}_{1}^{\uparrow(\downarrow)} \hat{\delta}+\hat{\delta} \hat{G}_{1}^{\downarrow(\uparrow)} \hat{\gamma} .
\end{aligned}
$$

One can regard $\hat{w}^{\uparrow(\downarrow)}$ as the effective spin-conserving potential, taking into account the effects of a disorder, and $\hat{J}_{ \pm}$- as the renormalized electron-magnon interaction. Finally, the simple algebra using (B3) (B7), yields

$$
\begin{aligned}
\hat{t}_{00} & =\langle 0|(\tilde{U}-\tilde{\Sigma}) \tilde{D}| 0\rangle \\
& =(\hat{W}-\hat{\Sigma})[1-\hat{G}(\hat{W}-\hat{\Sigma})]^{-1} \\
& =\left(\begin{array}{cc}
\hat{t}_{0}^{\uparrow} & \hat{t}_{0}^{+} \hat{S}_{-} \\
\hat{t}_{0}^{-} \hat{S}_{+} & \hat{t}_{0}^{\downarrow}
\end{array}\right) .
\end{aligned}
$$

Thus, we have obtained the logical result, that the $t$-matrix, averaged over configurations, corresponds to scattering on the effective potential $\hat{W}-\hat{\Sigma}$.

To evaluate the spin-conserving $\hat{t}_{0}^{\uparrow(\downarrow)}$ and spin-flip $\hat{t}_{0}^{ \pm}$ parts of scattering matrix, we introduce the propagators

$$
\hat{g}_{0}^{\uparrow(\downarrow)}=\left[1-\hat{G}^{\uparrow(\downarrow)}\left(\hat{w}^{\uparrow(\downarrow)}-\hat{\Sigma}^{\uparrow(\downarrow)}\right)\right]^{-1} \hat{G}^{\uparrow(\downarrow)},
$$

corresponding to the potentials $\hat{w}^{\uparrow \downarrow}-\Sigma^{\uparrow \downarrow}$ and define the "denominator"

$$
\hat{D}_{0}=[1-\hat{G}(\hat{W}-\hat{\Sigma})]^{-1}=\left(\begin{array}{cc}
\hat{D}_{0}^{\uparrow} & \hat{D}_{0}^{+} \\
\hat{D}_{0}^{-} & \hat{D}_{0}^{\downarrow}
\end{array}\right) .
$$

related with the effective potential $\hat{W}-\hat{\Sigma}$. The elements of $\hat{D}_{0}$ and propagator $\hat{G}_{0}$ can be expressed via $\hat{g}_{0}^{\uparrow(\downarrow)}$ using the form of potential $\hat{W}(\mathrm{~B} 8)$, namely

$$
\begin{aligned}
& \hat{G}_{0}^{\uparrow}=\left[1-\hat{G}^{\uparrow}\left(\hat{w}^{\uparrow}-\Sigma^{\uparrow}+\hat{J}_{+} \hat{n}_{+} \hat{g}_{0}^{\downarrow} \hat{J}_{-}\right)\right]^{-1} \hat{G}^{\uparrow}, \\
& \hat{G}_{0}^{\downarrow}=\left[1-\hat{G}^{\downarrow}\left(\hat{w}^{\downarrow}-\Sigma^{\downarrow}+\hat{J}_{-} \hat{n}_{-} \hat{g}_{0}^{\uparrow} \hat{J}_{+}\right)\right]^{-1} \hat{G}^{\downarrow}, \\
& \hat{G}_{0}^{+}=\hat{G}_{0}^{\uparrow} \hat{J}_{+} \hat{S}_{-} g_{0}^{\downarrow}, \quad \hat{G}_{0}^{-}=\hat{G}_{0}^{\downarrow} \hat{J}_{-} \hat{S}_{+} g_{0}^{\uparrow},
\end{aligned}
$$


and

$$
\begin{aligned}
& \hat{D}_{0}^{\uparrow}=\left[1-\hat{G}^{\uparrow}\left(\hat{w}^{\uparrow}-\hat{\Sigma}^{\uparrow}+\hat{J}_{+} \hat{n}_{+} \hat{g}_{0}^{\downarrow} \hat{J}_{-}\right)\right]^{-1}, \\
& \hat{D}_{0}^{\downarrow}=\left[1-\hat{G}^{\downarrow}\left(\hat{w}^{\downarrow}-\hat{\Sigma}^{\downarrow}+\hat{J}_{-} \hat{n}_{-} \hat{g}_{0}^{\uparrow} \hat{J}_{+}\right)\right]^{-1}, \\
& \hat{D}_{0}^{+}=\hat{G}_{0}^{\uparrow} \hat{J}_{+} \hat{S}_{-} d_{0}^{\downarrow}, \quad \hat{D}_{0}^{-}=\hat{G}_{0}^{\downarrow} \hat{J}_{-} \hat{S}_{+} d_{0}^{\uparrow},
\end{aligned}
$$

where "denominators" $d_{0}^{\uparrow(\downarrow)}$ are given by

$$
\tilde{d}_{0}^{\uparrow(\downarrow)}=\left[1-\hat{G}^{\uparrow(\downarrow)}\left(\hat{w}^{\uparrow(\downarrow)}-\hat{\Sigma}^{\uparrow(\downarrow)}\right)\right]^{-1} .
$$

Now, using (B12) and (B13) and taking into account the obvious relation $\hat{t}_{00}=(\hat{W}-\hat{\Sigma}) \hat{D}_{0}$, one finds

$$
\begin{aligned}
& \hat{t}_{0}^{\uparrow}=\left(\hat{w}^{\uparrow}-\hat{\Sigma}^{\uparrow}+\hat{J}_{+} \hat{n}_{+} \hat{g}_{0}^{\downarrow} \hat{J}_{-}\right) \hat{D}_{0}^{\uparrow}, \\
& \hat{t}_{0}^{\downarrow}=\left(\hat{w}^{\downarrow}-\hat{\Sigma}^{\downarrow}+\hat{J}_{-} \hat{n}_{-} \hat{g}_{0}^{\uparrow} \hat{J}_{+}\right) \hat{D}_{0}^{\downarrow}, \\
& \hat{t}_{0}^{+}=\hat{d}_{0}^{\uparrow T} \hat{J}_{+} \hat{D}_{0}^{\downarrow}, \quad \hat{t}_{0}^{-}=\hat{d}_{0}^{\downarrow T} \hat{J}_{-} \hat{D}_{0}^{\uparrow} .
\end{aligned}
$$

According to the ASF, $\hat{G}_{0}^{\uparrow(\downarrow)}$ and $\hat{t}_{0}^{\uparrow(\downarrow)}$ represent the configurationally averaged quantities, and after the averaging over magnon degrees of freedom one must get $\left\langle\hat{G}_{0}^{\uparrow(\downarrow)}\right\rangle_{b}=\hat{G}^{\uparrow(\downarrow)}$ and $\left\langle\hat{t}_{0}^{\uparrow(\downarrow)}\right\rangle_{b}=0$. It means that due to the CPA self-consistency conditions the averaged scattering $t$-matrix must vanish and averaged electron propagator has to be equal to the effective Green's function (21), determined by the self-energy operator $\hat{\Sigma}$. Carrying out the averaging procedure over magnon degrees of freedom the similar way as it was proposed earlier in Sec. IIC, it is possible to satisfy both of these condition if one assumes that

$$
\begin{aligned}
& \hat{\Sigma}^{\uparrow}=\hat{\omega}^{\uparrow}(n)+\hat{J}_{+}(n) n \hat{g}_{0}^{\downarrow}(n) \hat{J}_{-}(n), \\
& \hat{\Sigma}^{\downarrow}=\hat{\omega}^{\downarrow}(n)+\hat{J}_{-}(n) n \hat{g}_{0}^{\uparrow}(n) \hat{J}_{+}(n) .
\end{aligned}
$$

We have pointed out the explicit dependence of matrices $\hat{\omega}^{\uparrow(\downarrow)}(n), \hat{J}_{ \pm}(n)$ and $\hat{g}_{0}^{\uparrow(\downarrow)}(n)$ on the average magnon number $n$ (Eq. 33). This dependence is assumed to be the same as it comes from the initial definition of operators $\hat{\omega}^{\uparrow(\downarrow)}, \hat{J}_{ \pm}$, and $\hat{g}_{0}^{\uparrow(\downarrow)}$ as functions on $\hat{n}_{ \pm}$[Eqs. (B9)(B11)], that is the consequence of the adopted approximate averaging procedure. The matrices $\hat{\omega}^{\uparrow(\downarrow)}, \hat{J}_{ \pm}$and $\hat{g}_{0}^{\uparrow(\downarrow)}$ functionally depend on $\Sigma^{\uparrow(\downarrow)}$. Due to that, the system (B15) represents the alternative to Eq. (34) form of the $\mathrm{CPA}$ conditions and it can be simply solved by means of successive numerical iterations.
1 M. Julliere, Phys. Lett. A 54, 225 (1975)

2 J. S. Moodera, L. R. Kinder, T. M. Wong, and R. Meservey, Phys. Rev. Lett. 74, 3273 (1995); J. S. Moodera, L. R. Kinder, J. Appl. Phys. 79, 4724 (1996); J. S. Moodera, J. Nowak, and R. J. M. van de Veerdonk, Phys. Rev. Lett. 80, 2941 (1998)

3 T. Miyazaki, N. Tezuka, Journ. Magn. Magn. Mater. 139, L231 (1995); N. Tezuka and T. Miyazaki, J. Appl. Phys. 79, 6262 (1996); T. Miyazaki, S. Kumagai, T. Yaoi, J. Appl. Phys. 81, 3753 (1997)

${ }^{4}$ W. J. Gallagher, S. S. P. Parkin, Yu Lu, X. P. Bian, A. Marley, K. P. Roche, R. A. Altman, S. A. Rishton, C. Jahnes, T. W. Shaw, and G. Xiao, J. Appl. Phys. 81, 3741 (1997)

5 R. Meservey and P. M. Tedrow, Phys. Rep. 238, 173 (1994)

${ }^{6}$ S. Zhang and P. M. Levy, Eur. Phys. J. B 10, 599 (1999)

7 J. S. Moodera and G. Mathon, J. Magn. Magn. Mater. 200, 248 (1999)

8 J. S. Moodera, T. H. Kim, C. Tanaka, and C. H. de Groot, Phil. Mag. B 80, 195 (2000)

9 J. S. Moodera, E. F. Gallagher, K. Robinson, and J. Nowak, Appl. Phys. Lett. 70, 3050 (1997)

10 T. Mitsuzuka, K. Matsuda, A. Kamijo, and H. Tsuge, J. Appl. Phys. 85, 5807 (1999)

11 J. M. MacLaren, X. G. Zhang, and W. H. Butler, Phys. Rev. B 56, 11827 (1997)

12 J. C. Slonczewski, Phys. Rev. B 39, 6995 (1989);

13 S. Zhang, P. M. Levy, A. C. Marley, and S. S. P. Parkin, Phys. Rev. Lett. 79, 3744 (1997)

14 E. Yu. Tsymbal and D. G. Pettifor, J. Phys.: Cond. Matt. 9, L411 (1997)
15 J. Mathon, Phys. Rev. B 56, 11810 (1997)

16 W. H. Butler, X. G. Zhang, X. Wang, J. V. Ek, and J. M. MacLaren, J. Appl. Phys. 81, 5518 (1997); J. M. MacLaren, W. H. Butler, and X. G. Zhang, J. Appl. Phys. 81, 6521 (1998)

17 I. I. Oleinik, E. Yu. Tsymbal, and D. G. Pettifor, Phys. Rev. B 62, 3952 (2000)

18 J. M. MacLaren, X. G. Zhang, W. H. Butler, and X. Wang, Phys. Rev. B 59, 5470 (1999)

19 W. H. Butler, X. G. Zhang, T. C. Schulthess, and J. M. MacLaren, Phys. Rev. B 63, 054416 (2001)

20 J. Mathon and A. Umerski, Phys. Rev. B 63, 220403(R) (2001)

21 T. L. Monchesky, A. Enders, R. Urban, J. F. Cochran, B. Heinrich, W. Wulfhekel, M. Klaua, F. Zavaliche, and J. Kirschner, in The Physics of Low Dimensions, edited by Jose Luis Moran Lopez (Plenum, New York, 2000)

22 O. Wunnicke, N. Papanikolaou, R. Zeller, P. H. Dederichs, V. Drchal, and J. Kudrnovsky, communication on the European Graduate School on Condensed Matter (EGSCM Prague'01) (unpublished); M. Zwierzycki, K. Xia, P. H. Kelly, G. E. Bauer, I. Turek, ibid. (unpublished)

23 A. Vedyayev, N. Ryzhanova, R. Vlutters, and B. Dieny, Europhys. Lett. 46, 808 (1998)

24 P. Soven, Phys. Rev. 156, 809 (1967)

25 B. Velicky, Phys. Rev. 184, 614 (1969)

${ }^{26}$ V. L. Moruzzi, J. F. Janak, and A. R. Williams, Calculated Electronic Properties of Metals (Pergamon, New York, 1978).

27 M. B. Stearns, J. Magn. Magn. Mater. 5, 167 (1977)

28 D. A. Papaconstantopoulos, Handbook of the Band Struc- 
ture of Elemental Solids (Plenum, New York, 1986)

${ }^{29}$ Y. N. Xu, W. Y. Ching, Phys. Rev. B 43, 4461 (1991)

${ }^{30}$ P. LeClair, J. T. Kohlhepp, H. J. M. Swagten, and W. J. M. de Jonge, Phys. Rev. Lett. 86, 1066 (2001)

31 P. LeClair, H. J. M. Swagten, J. T. Kohlhepp, R. J. M. van de Veerdonk, and W. J. M. de Jonge, Phys. Rev. Lett. 84, 2933 (2000)

32 R. Kubo, M. Toda and N. Hashitsume. Statistical Physics II. Nonequilibrium Statistical Mechanics. (Springer, Berlin, 1985)

33 A. Vedyayev, N. Ryzhanova, C. Lacroix, L. Giacomoni, and B. Dieny, Europhys. Lett. 39, 219 (1997)

34 P. M. Levy, H. E. Camblong, and S. Zhang, J. Appl. Phys. 75, 7076 (1994); P. M. Levy, Solid State Physics 47, 367 (1994)

35 H. Ehrenreich, Solid State Physics 31, 149 (1976)

36 A. Mookerjee, J. Phys. C 6, L205, 1340 (1973)

37 V. Heine, Phys. Rev. 153, 673 (1966)

$38 \mathrm{Yu} \mathrm{Lu}$, X. W. Li, Gang Xiao, R. A. Altman, W. J. Gallagher, A. Marley, K. Roche, S. Parkin, J. Appl. Phys. 83, 6515 (1998) 